MATHEMATICS OF COMPUTATION

Volume 66, Number 217, January 1997, Pages 157-192

S 0025-5718(97)00776-X

\title{
A STOCHASTIC PARTICLE METHOD FOR THE MCKEAN-VLASOV AND THE BURGERS EQUATION
}

\author{
MIREILLE BOSSY AND DENIS TALAY
}

\begin{abstract}
In this paper we introduce and analyze a stochastic particle method for the McKean-Vlasov and the Burgers equation; the construction and error analysis are based upon the theory of the propagation of chaos for interacting particle systems.

Our objective is three-fold. First, we consider a McKean-Vlasov equation in $[0, T] \times \mathbb{R}$ with sufficiently smooth kernels, and the PDEs giving the distribution function and the density of the measure $\mu_{t}$, the solution to the McKean-Vlasov equation. The simulation of the stochastic system with $N$ particles provides a discrete measure which approximates $\mu_{k \Delta t}$ for each time $k \Delta t$ (where $\Delta t$ is a discretization step of the time interval $[0, T]$ ). An integration (resp. smoothing) of this discrete measure provides approximations of the distribution function (resp. density) of $\mu_{k \Delta t}$. We show that the convergence rate is $\mathcal{O}(1 / \sqrt{N}+\sqrt{\Delta t})$ for the approximation in $L^{1}(\Omega \times \mathbb{R})$ of the cumulative distribution function at time $T$, and of order $\mathcal{O}\left(\varepsilon^{2}+\frac{1}{\varepsilon}\left(\frac{1}{\sqrt{N}}+\sqrt{\Delta t}\right)\right)$ for the approximation in $L^{1}(\Omega \times \mathbb{R})$ of the density at time $T$ ( $\Omega$ is the underlying probability space, $\varepsilon$ is a smoothing parameter). Our second objective is to show that our particle method can be modified to solve the Burgers equation with a nonmonotonic initial condition, without modifying the convergence rate $\mathcal{O}(1 / \sqrt{N}+\sqrt{\Delta t})$. This part extends earlier work of ours, where we have limited ourselves to monotonic initial conditions. Finally, we present numerical experiments which confirm our theoretical estimates and illustrate the numerical efficiency of the method when the viscosity coefficient is very small.
\end{abstract}

\section{INTRODUCTION}

Consider two Lipschitz kernels $b(x, y), s(x, y)$ from $\mathbb{R}^{2}$ to $\mathbb{R}$, a probability measure $\mu_{0}$ and the differential operator $L(\mu)$ defined by

$$
L(\mu) f(x)=\frac{1}{2}\left(\int_{\mathbb{R}} s(x, y) d \mu(y)\right)^{2} f^{\prime \prime}(x)+\left(\int_{\mathbb{R}} b(x, y) d \mu(y)\right) f^{\prime}(x) .
$$

Consider the probability measure $\mu_{t}$, the solution to the McKean-Vlasov equation

$$
\left\{\begin{array}{l}
\frac{d}{d t}\left\langle\mu_{t}, f\right\rangle=\left\langle\mu_{t}, L\left(\mu_{t}\right) f\right\rangle, \forall f \in C_{K}^{\infty}(\mathbb{R}) \\
\mu_{t=0}=\mu_{0}
\end{array}\right.
$$

Received by the editor January 11, 1995 and, in revised form, May 15, 1995 and November 6, 1995.

1991 Mathematics Subject Classification. Primary 60H10, 60K35, 65C20, 65M15, 65U05.

Key words and phrases. Stochastic particle methods, interacting particle systems, Burgers equation.

(C)1997 American Mathematical Society 
References concerning this equation and its connection with Physics can be found in Gärtner [9].

The distribution function $V(t, x)$ of $\mu_{t}$ solves

$$
\left\{\begin{aligned}
& \frac{\partial V}{\partial t}(t, x)=\frac{1}{2} \frac{\partial}{\partial x}\left[\left(\int_{\mathbb{R}} s(x, y) \frac{\partial V}{\partial x}(t, y) d y\right)^{2} \frac{\partial V}{\partial x}(t, x)\right]-\left[\int_{\mathbb{R}} b(x, y) \frac{\partial V}{\partial x}(t, y) d y\right] \frac{\partial V}{\partial x}(t, x), \\
& V(0, x)=V_{0}(x)
\end{aligned}\right.
$$

where $V_{0}(\cdot)$ is the distribution function of $\mu_{0}$.

Under suitable hypotheses on $b(x, y), s(x, y)$, for each $t>0$ the measure $\mu_{t}$ has a smooth density $u(t, \cdot)$, which is the classical solution to

$$
\left\{\begin{array}{c}
\frac{\partial u}{\partial t}(t, x)=\frac{1}{2} \frac{\partial^{2}}{\partial x^{2}}\left[u(t, x)\left(\int_{\mathbb{R}} s(x, y) u(t, y) d y\right)^{2}\right] \\
-\frac{\partial}{\partial x}\left[u(t, x) \int_{\mathbb{R}} b(x, y) u(t, y) d y\right], \\
u(0, x)=u_{0}(x),
\end{array}\right.
$$

where $u_{0}(\cdot)$ is the density of $\mu_{0}$.

In this paper, we construct an approximation method for the solutions of (1.1), (1.2) and (1.3) and give its convergence rate. Our analysis is based upon the following probabilistic interpretation. Consider the discrete measure

$$
\mu_{t}^{N}=\frac{1}{N} \sum_{i=1}^{N} \delta_{X_{t}^{i, N}}
$$

where the $X_{t}^{i, N}$ 's $(1 \leq i \leq N)$ denote the locations at time $t$ of a suitable weakly interacting stochastic particle system; the PDE (1.1) describes the time evolution of the weak limit of the empirical measure $\mu_{t}^{N}$ when the number of particles goes to infinity. Likewise, the corresponding distribution functions $\left(V_{t}^{N}(\cdot)\right)$ converge pointwise to the solution $V(t, \cdot)$ of the PDE (1.2).

In practice, the $X_{t}^{i, N}$, s cannot be computed exactly; our algorithm involves their approximation by a discrete-time stochastic process $\left(\bar{X}_{k \Delta t}^{i}, 1 \leq i \leq N\right)$, where $\Delta t$ is a discretization step of the time interval $[0, T]$; we denote by $\bar{\mu}_{k \Delta t}^{N, \bar{\Delta} t}$ the empirical measure

$$
\bar{\mu}_{k \Delta t}^{N, \Delta t}:=\frac{1}{N} \sum_{i=1}^{N} \delta_{\bar{X}_{k \Delta t}^{i}} ;
$$

the distribution function of $\bar{\mu}_{k \Delta t}^{N, \Delta t}$ is denoted by $\bar{V}_{k \Delta t}^{N, \Delta t}$.

We first analyze the convergence rate of $\bar{V}_{T}^{N, \Delta t}$ to $V(T, \cdot)$ when the kernels $b$ and $s$ are bounded functions with bounded first derivatives, and $s$ is bounded below by a strictly positive constant. Under these hypotheses, $\mu_{t}$ for all $t>0$ has a density $u(t, \cdot)$ with respect to Lebesgue measure. We show

$$
\mathbb{E}\left\|V(T, \cdot)-\bar{V}_{T}^{N, \Delta t}(\cdot)\right\|_{L^{1}(\mathbb{R})}=\mathcal{O}\left(\frac{1}{\sqrt{N}}+\sqrt{\Delta t}\right)
$$

and

$$
\mathbb{E}\left\|u(T, \cdot)-\bar{u}_{T}^{N, \Delta t, \varepsilon}(\cdot)\right\|_{L^{1}(\mathbb{R})}=\mathcal{O}\left(\varepsilon^{2}+\frac{1}{\varepsilon}\left(\frac{1}{\sqrt{N}}+\sqrt{\Delta t}\right)\right),
$$

where $\bar{u}_{T}^{N, \Delta t, \varepsilon}$ denotes a smoothing of $\bar{\mu}^{N, \Delta t}$. 
Now consider a constant kernel $s(x, y)$ equal to $\sigma$, and instead of choosing a Lipschitz kernel $b$, choose $b(x, y)=H(x-y)$, where $H$ is the Heaviside function $(H(z)=1$ if $z \geq 0, H(z)=0$ if $z<0)$; then the equation (1.2) becomes the Burgers equation

$$
\left\{\begin{array}{l}
\frac{\partial V}{\partial t}(t, x)=\frac{1}{2} \sigma^{2} \frac{\partial^{2} V}{\partial x^{2}}(t, x)-V(t, x) \frac{\partial V}{\partial x}(t, x) \text { in }[0, T] \times \mathbb{R} \\
V(0, x)=V_{0}(x)
\end{array}\right.
$$

The corresponding analysis of

$$
\mathbb{E}\left\|V(T, \cdot)-\bar{V}_{T}^{N, \Delta t}(\cdot)\right\|_{L^{1}(\mathbb{R})}
$$

is made in Bossy and Talay [3] under the hypothesis that $V_{0}$ is the distribution function of a probability measure: we prove that (1.4) also holds in spite of the discontinuity at the origin of the Heaviside function. As this discontinuity generates technical difficulties and the study of a nonlinear martingale problem, a reader unfamiliar with the stochastic calculus should first read the present paper, even though in other respects some key arguments are similar in the Lipschitz and the Heaviside situations.

The second objective of this article is to extend our approach and our theoretical estimate to the Burgers equation when the initial condition $V_{0}$ is not a distribution function: we prove a suitable probabilistic interpretation of the solution, which suggests a slight modification of our stochastic particle method; the convergence rate (1.4) is preserved. This work is done for the Burgers equation because this case is technically more complex than the situation of Lipschitz kernels, but the algorithmic principle and the error analysis would also apply in this latter situation.

The last objective of the paper is to present numerical results which illustrate the following points: our theoretical estimates are optimal with respect to $N$, and our stochastic particle method efficiently solves the Burgers equation even when the viscosity coefficient $\sigma$ is very small.

Another stochastic particle method for the Burgers equation has been proposed by Roberts [27]; his algorithm is based on the splitting of the nonlinear operator, similarly to the well-known random vortex methods for the incompressible $2 \mathrm{D}$ Navier-Stokes equation developed by Chorin, Hald, etc. (Chorin [6], Chorin and Marsden [5], Goodman [10], Hald ([12] and [13]), Long [18], Puckett [24] e.g.; see also the bibliography in [6] and in the different contributions of [11]. A stochastic particle method for convection-reaction-diffusion equations with a nonlinear reaction term has been proposed by Puckett [25], and its convergence rate has been analyzed by Bernard, Talay and Tubaro [1]).

The novelty of our approach consists in ignoring the splitting: the particle method is interpreted through the link between the nonlinear PDE and systems of interacting particles. Furthermore, we emphasize that the choice of the Heaviside function for the interaction kernel $b$ is not the only one possible for the Burgers equation. One can also interpret the Burgers equation as the Fokker-Planck equation for the limit law of a particle system corresponding to a kernel $b$, roughly speaking, equal to a Dirac measure (see Sznitman [28]). The corresponding algorithm must involve a smoothing of this kernel. The numerical analysis is made complex by a necessary relation between the smoothing parameter and the number of particles: see Bossy [2] for a discussion. 
In $\S 2$ we prove the estimates (1.4) and (1.5). In $\S 3$ we establish a probabilistic interpretation of the solution of the Burgers equation with a not necessarily monotonic initial condition: this new interpretation leads to a stochastic particle method for which the estimate (1.4) still holds. In $\S 4$ we present the results of numerical experiments. Our main results are stated in the subsections 2.2 and 3.4.

\section{MCKean-Vlasov Equations With SMOOTH KERnELS}

Consider the system of stochastic differential equations describing the dynamics of weakly interacting particles:

$$
\left\{\begin{array}{l}
d X_{t}^{i, N}=\int_{\mathbb{R}} b\left(X_{t}^{i, N}, y\right) \mu_{t}^{N}(d y) d t+\int_{\mathbb{R}} s\left(X_{t}^{i, N}, y\right) \mu_{t}^{N}(d y) d w_{t}^{i} \\
X_{0}^{i, N}=X_{0}^{i}, i=1, \ldots, N
\end{array}\right.
$$

where $\left(w_{t}^{1}\right), \ldots,\left(w_{t}^{N}\right)$ are independent one-dimensional Brownian motions and $\mu_{t}^{N}$ is the random empirical measure

$$
\mu_{t}^{N}=\frac{1}{N} \sum_{i=1}^{N} \delta_{X_{t}^{i, N}}
$$

The functions $b$ and $s$ are the "interaction kernels". When the initial distribution of the particles is symmetric, and when the kernels are Lipschitz, the propagation of chaos theory shows that the random probability measure

$$
\mu^{N}=\frac{1}{N} \sum_{i=1}^{N} \delta_{X^{i, N}}
$$

on the space of trajectories converges in law, as $N$ goes to infinity, to a deterministic probability measure $\mu$ (see Sznitman [28] for a review, Gärtner [9], Léonard [17], Méléard and Roelly [20], Métivier [21], Oelschläger [23] for other types of interaction). For each $t$, denote by $\mu_{t}$ the one-dimensional distribution of $\mu$ ( $\mu_{t}$ is the limit in law of $\left.\mu_{t}^{N}\right)$. There exists a unique strong solution $\left(X_{t}\right)$ to the nonlinear stochastic differential equation (see Sznitman [28] e.g.)

$$
\left\{\begin{array}{l}
X_{t}=X_{0}+\int_{0}^{t} \int_{\mathbb{R}} b\left(X_{\theta}, y\right) \mu_{\theta}(d y) d \theta+\int_{0}^{t} \int_{\mathbb{R}} s\left(X_{\theta}, y\right) \mu_{\theta}(d y) d w_{\theta}, \\
\mu_{t} \text { is the law of the random variable } X_{t}, \text { for all } t \geq 0 .
\end{array}\right.
$$

One consequence of the propagation of chaos is that the law of one particle, for example the law of $\left(X_{t}^{1, N}\right)$, tends to the law of the process $\left(X_{t}\right)$ when $N$ goes to infinity.

Applying Itô's formula, one deduces from (2.2) that $\mu_{t}$ is the solution to (1.1).

We suppose that the following assumptions hold:

(H1) There exists a strictly positive constant $s_{*}$ such that $s(x, y) \geq s_{*}>0, \forall(x, y)$.

(H2) The kernels $b$ and $s$ are uniformly bounded functions on $\mathbb{R}^{2} ; b$ is globally Lipschitz and $s$ has uniformly bounded first partial derivatives.

(H3) The initial law $\mu_{0}$ satisfies:

(i) either $\mu_{0}$ is a Dirac measure at $x_{0}$, or 
(ii) $\mu_{0}$ has a continuous density $u_{0}$ satisfying: there exist constants $M>0$, $\eta \geq 0$ and $\alpha>0$ such that $u_{0}(x) \leq \eta \exp \left(-\alpha \frac{x^{2}}{2}\right)$ for $|x|>M$.

(If $\eta=0, \mu_{0}$ has compact support.)

2.1. The algorithm. In this subsection we construct an approximation method for the solutions of (1.2) and (1.3), based upon the time discretization of the system (2.1). From now on, the number $N$ of particles is fixed.

The algorithm starts with an approximation of the initial condition $V(0, \cdot)=$ $V_{0}(\cdot)$, the distribution function of the law $\mu_{0}$. The $N$ points $\left(y_{0}^{1}, \ldots, y_{0}^{N}\right)$ are chosen in $\mathbb{R}$ such that the piecewise constant function

$$
\bar{V}_{0}(x)=\frac{1}{N} \sum_{i=1}^{N} H\left(x-y_{0}^{i}\right)
$$

approximates $V_{0}$ in $L^{1}(\mathbb{R})$ with a sufficiently high accuracy. As we will see, a possible choice is the following: if $\mu_{0}$ is a Dirac measure at $x_{0}$, the $N$ particles are located at $y_{0}^{i}=x_{0}$ (and then $\bar{V}_{0}(\cdot)=V_{0}(\cdot)$ ); when $\mu_{0}$ satisfies (H3-ii), we set

$$
y_{0}^{i}=\left\{\begin{array}{l}
\inf \left\{y ; V_{0}(y)=\frac{i}{N}\right\}, i=1, \ldots, N-1, \\
\inf \left\{y ; V_{0}(y)=1-\frac{1}{2 N}\right\} .
\end{array}\right.
$$

We set

$$
\bar{\mu}_{0}:=\frac{1}{N} \sum_{i=1}^{N} \delta_{y_{0}^{i}} .
$$

Consider the system (2.1) with the initial condition $X_{0}^{i, N}=y_{0}^{i}$, and denote its solution by $\left(X_{t}^{i}, 1 \leq i \leq N\right)$. There holds

$$
\left\{\begin{array}{l}
d X_{t}^{i}=\frac{1}{N} \sum_{j=1}^{N} b\left(X_{t}^{i}, X_{t}^{j}\right) d t+\frac{1}{N} \sum_{j=1}^{N} s\left(X_{t}^{i}, X_{t}^{j}\right) d w_{t}^{i}, t \in[0, T], \\
X_{0}^{i}=y_{0}^{i}, i=1, \ldots, N
\end{array}\right.
$$

We recall that the propagation of chaos suggests that $1 / N \sum_{i=1}^{N} \delta_{X_{t}^{i}}$ approximates the solution $\mu_{t}$ of (1.1).

To get a simulation procedure for a trajectory of each $\left(X_{t}^{i}\right)$, we discretize in time: $\Delta t>0$ and $K \in \mathbb{N}$ are chosen such that $T=K \Delta t$; the discrete times are denoted by $t_{k}=k \Delta t$, with $1 \leq k \leq K$. The Euler scheme leads to the following discrete-time system:

$$
\left\{\begin{array}{l}
Y_{t_{k+1}}^{i}=Y_{t_{k}}^{i}+\frac{1}{N} \sum_{j=1}^{N} b\left(Y_{t_{k}}^{i}, Y_{t_{k}}^{j}\right) \Delta t+\frac{1}{N} \sum_{j=1}^{N} s\left(Y_{t_{k}}^{i}, Y_{t_{k}}^{j}\right)\left(w_{t_{k+1}}^{i}-w_{t_{k}}^{i}\right), \\
Y_{0}^{i}=y_{0}^{i}, i=1, \ldots, N .
\end{array}\right.
$$

Thus, we approximate $\mu_{t_{k}}$ by the empirical measure

$$
\bar{\mu}_{t_{k}}:=\frac{1}{N} \sum_{i=1}^{N} \delta_{Y_{t_{k}}^{i}} .
$$


In the same way, we approximate $V\left(t_{k}, \cdot\right)$, solution to $(1.2)$, by the cumulative distribution function of $\mu_{t_{k}}$ :

$$
\bar{V}_{t_{k}}(x):=\frac{1}{N} \sum_{i=1}^{N} H\left(x-Y_{t_{k}}^{i}\right), \forall x \in \mathbb{R} .
$$

Note that we have changed the notation of the introduction: $Y_{t_{k}}^{i}$ corresponds to $\bar{X}_{k \Delta t}^{i}$ and $\bar{\mu}_{t_{k}}$ stands for $\bar{\mu}_{t_{k}}^{N, \Delta t}$.

2.2. Main results. In the next subsection we will prove the following two theorems.

Theorem 2.1. Assume (H1), (H2) and (H3). Let $V(t, x)$ be the solution of the $P D E$ (1.2). For $T>0$ fixed, let $\Delta t<1$ be such that $T=\Delta t K, K \in \mathbb{N}$. Let $\bar{V}_{t_{k}}(x)$ be the approximation corresponding to the above algorithm with $N$ particles. Then there exist strictly positive constants $L_{1}$ and $L_{2}$ depending on $s, b, V_{0}$ and $T$ such that for all $k \in\{1, \ldots, K\}$ one has

$$
\mathbb{E}\left\|V\left(t_{k}, \cdot\right)-\bar{V}_{t_{k}}(\cdot)\right\|_{L^{1}(\mathbb{R})} \leq L_{1}\left(\left\|V_{0}-\bar{V}_{0}\right\|_{L^{1}(\mathbb{R})}+\frac{1}{\sqrt{N}}+\sqrt{\Delta t}\right)
$$

and

$$
\operatorname{Var}\left(\left\|V\left(t_{k}, \cdot\right)-\bar{V}_{t_{k}}(\cdot)\right\|_{L^{1}(\mathbb{R})}\right) \leq L_{2}\left(\left\|V_{0}-\bar{V}_{0}\right\|_{L^{1}(\mathbb{R})}^{2}+\frac{1}{N}+\Delta t\right) .
$$

Furthermore,

$$
\left\|V_{0}-\bar{V}_{0}\right\|_{L^{1}(\mathbb{R})} \leq \frac{C \sqrt{\log (N)}}{N}
$$

where $C$ depends on $M, \eta$ and $\alpha$.

As we will see, (H1) and (H2) imply that for all $t>0$ the measure $\mu_{t}$ has a density $u(t, \cdot)$ with respect to Lebesgue measure. In order to obtain an approximation of $u\left(t_{k}, \cdot\right)$, we construct a regularization by convolution of the discrete measure $\bar{\mu}_{t_{k}}$.

Let $\Phi_{\varepsilon}$ be the density of the Gaussian law $N\left(0, \varepsilon^{2}\right)$ and set

$$
\bar{u}_{t_{k}}^{\varepsilon}(x):=\left(\Phi_{\varepsilon} * \bar{\mu}_{t_{k}}\right)(x)=\frac{1}{N} \sum_{i=1}^{N} \frac{1}{\sqrt{2 \pi} \varepsilon} \exp \left(-\frac{\left(x-Y_{t_{k}}^{i}\right)^{2}}{2 \varepsilon^{2}}\right) .
$$

We strengthen our hypotheses as follows:

$\left(\mathrm{H} 2^{\prime}\right)$ The kernel $b$ is in $C_{b}^{2}\left(\mathbb{R}^{2}\right)$ and $s$ is in $C_{b}^{3}\left(\mathbb{R}^{2}\right)$.

$\left(\mathrm{H} 3^{\prime}\right)$ The initial law $\mu_{0}$ has a strictly positive density $u_{0}$ in $C^{2}(\mathbb{R})$ satisfying: there exist strictly positive constants $M, \eta$ and $\alpha$ such that

$$
u_{0}(x)+\left|u_{0}^{\prime}(x)\right|+\left|u_{0}^{\prime \prime}(x)\right| \leq \eta \exp \left(-\alpha \frac{x^{2}}{2}\right) \text { for }|x|>M .
$$

Theorem 2.2. Assume $(\mathrm{H} 1),\left(\mathrm{H} 2^{\prime}\right)$ and $\left(\mathrm{H} 3^{\prime}\right)$. Let $u(t, \cdot)$ be the classical solution to the PDE (1.3). Then there exists a strictly positive constant $C$, depending on $s$, $b, u_{0}$ and $T$, such that for all $k \in\{1, \ldots, K\}$ one has

$$
\mathbb{E}\left\|u\left(t_{k}, \cdot\right)-\bar{u}_{t_{k}}^{\varepsilon}(\cdot)\right\|_{L^{1}(\mathbb{R})} \leq C\left[\varepsilon^{2}+\frac{1}{\varepsilon}\left(\left\|V_{0}-\bar{V}_{0}\right\|_{L^{1}(\mathbb{R})}+\frac{1}{\sqrt{N}}+\sqrt{\Delta t}\right)\right]
$$


and

$$
\operatorname{Var}\left(\left\|u\left(t_{k}, \cdot\right)-\bar{u}_{t_{k}}^{\varepsilon}(\cdot)\right\|_{L^{1}(\mathbb{R})}\right) \leq C\left[\varepsilon^{4}+\frac{1}{\varepsilon^{2}}\left(\left\|V_{0}-\bar{V}_{0}\right\|_{L^{1}(\mathbb{R})}^{2}+\frac{1}{N}+\Delta t\right)\right] .
$$

Remark 2.3. The hypothesis (H1) could be somewhat relaxed: what is used in the proof is the existence of a density for the law of the process $\left(z_{t}\right)$ defined below in (2.6), this density satisfying the exponential bounds (2.11) below.

2.3. Proof of Theorem 2.1. Throughout this section, $C$ denotes any positive constant depending only on $T, V_{0}$ and the functions $b, s$.

Before entering the technical parts of the rather lengthy proof, we present a sketch of it.

2.3.1. Decomposition of the error and sketch of the proof. The proof of Theorem 2.1 is based upon a decomposition of the error $\mathbb{E}\left\|V\left(t_{k}, .\right)-\bar{V}_{t_{k}}(\cdot)\right\|_{L^{1}(\mathbb{R})}$ at each time $t_{k}$ of the discretization: this decomposition corresponds to local errors of different natures in the algorithm.

Define $\beta:[0, T] \times \mathbb{R} \longrightarrow \mathbb{R}$ by

$$
\beta(t, x):=\int_{\mathbb{R}} b(x, y) \mu_{t}(d y),
$$

and $\sigma:[0, T] \times \mathbb{R} \longrightarrow \mathbb{R}$ by

$$
\sigma(t, x):=\int_{\mathbb{R}} s(x, y) \mu_{t}(d y) .
$$

Under (H2), $\beta$ and $\sigma$ are Lipschitz in $x$ and uniformly bounded in $(t, x)$; this ensures the strong existence and uniqueness of the inhomogeneous Markov process solution to the stochastic differential equation

$$
\left\{\begin{array}{l}
d z_{t}=\beta\left(t, z_{t}\right) d t+\sigma\left(t, z_{t}\right) d w_{t}, \\
z_{t=0}=z_{0},
\end{array}\right.
$$

where $z_{0}$ is a square integrable random variable. When the law of $z_{0}$ is $\mu_{0}$, the two processes $\left(z_{t}\right)$ and $\left(X_{t}\right)$, solution to $(2.2)$, have the same law and

$$
V(t, x)=\mathbb{E} H\left(x-X_{t}\right)=\mathbb{E}_{\mu_{0}} H\left(x-z_{t}\right)=\int_{\mathbb{R}} \mathbb{E} H\left(x-z_{t}(y)\right) \mu_{0}(d y),
$$

where $\left(z_{t}(y)\right)$ is the solution to $(2.6)$ starting at $y$ at time 0 . Note that $\left(z_{t}\right)$ is a Markov process, whereas $\left(X_{t}\right)$ is not: this is used in the sequel.

A first approximation of $V(t, \cdot)$ is given by

$$
V(t, x) \simeq \mathbb{E}_{\bar{\mu}_{0}} H\left(x-z_{t}\right)=\frac{1}{N} \sum_{i=1}^{N} \mathbb{E} H\left(x-z_{t}\left(y_{0}^{i}\right)\right) .
$$

Now consider $N$ independent Brownian motions $\left(w_{t}^{i}\right)_{i=1}^{N}$ and the family of independent processes $\left(z_{t}^{i}\right)_{(i=1, \ldots, N)}$, solutions to

$$
\left\{\begin{array}{l}
d z_{t}^{i}=\beta\left(t, z_{t}^{i}\right) d t+\sigma\left(t, z_{t}^{i}\right) d w_{t}^{i}, \\
z_{0}^{i}=y_{0}^{i} .
\end{array}\right.
$$


A new approximation of $V(t, \cdot)$ is given by

$$
V(t, x) \simeq \frac{1}{N} \sum_{i=1}^{N} H\left(x-z_{t}^{i}\right)
$$

Applying the Euler scheme to (2.7), one defines the independent discrete-time processes $\left(\bar{z}_{t_{k}}^{i}\right)$ :

$$
\left\{\begin{array}{l}
\bar{z}_{t_{k+1}}^{i}=\bar{z}_{t_{k}}^{i}+\beta\left(t_{k}, \bar{z}_{t_{k}}^{i}\right) \Delta t+\sigma\left(t_{k}, \bar{z}_{t_{k}}^{i}\right)\left(w_{t_{k+1}}^{i}-w_{t_{k}}^{i}\right), \\
\bar{z}_{0}^{i}=y_{0}^{i} .
\end{array}\right.
$$

Thus, at time $t_{k}$ (with $\left.k=1, \ldots, K\right), V\left(t_{k}, \cdot\right)$ can be approximated by

$$
V\left(t_{k}, x\right) \simeq \frac{1}{N} \sum_{i=1}^{N} H\left(x-z_{t_{k}}^{i}\right) .
$$

This latter approximation is not useful from a numerical point of view since the functions $\beta(t, \cdot)$ and $\sigma(t, \cdot)$ are unknown (they are defined through the unknown measure $\left.\mu_{t}\right)$. We therefore substitute the empirical approximation to $\mu_{t}$, which leads to the system (2.3); in other words, we make the additional approximations

$$
\begin{aligned}
& \beta\left(t_{k}, x\right)=\int_{\mathbb{R}} b(x, y) \mu_{t_{k}}(d y) \simeq \int_{\mathbb{R}} b(x, y) \bar{\mu}_{t_{k}}(d y)=\frac{1}{N} \sum_{i=1}^{N} b\left(x, Y_{t_{k}}^{i}\right), \\
& \sigma\left(t_{k}, x\right) \simeq \frac{1}{N} \sum_{i=1}^{N} s\left(x, Y_{t_{k}}^{i}\right) .
\end{aligned}
$$

Finally, the algorithm consists in approximating the function $V\left(t_{k}, x\right)$ by

$$
\bar{V}_{t_{k}}(x)=\frac{1}{N} \sum_{i=1}^{N} H\left(x-Y_{t_{k}}^{i}\right) .
$$

The preceding considerations suggest the following decomposition of the global error:

$$
\begin{aligned}
& \mathbb{E}\left\|V\left(t_{k}, x\right)-\bar{V}_{t_{k}}(x)\right\|_{L^{1}(\mathbb{R})} \\
& \quad \leq\left\|\mathbb{E}_{\mu_{0}} H\left(x-z_{t_{k}}\right)-\mathbb{E}_{\bar{\mu}_{0}} H\left(x-z_{t_{k}}\right)\right\|_{L^{1}(\mathbb{R})} \\
& \quad+\mathbb{E}\left\|\mathbb{E}_{\bar{\mu}_{0}} H\left(x-z_{t_{k}}\right)-\frac{1}{N} \sum_{i=1}^{N} H\left(x-z_{t_{k}}^{i}\right)\right\|_{L^{1}(\mathbb{R})} \\
& \quad+\mathbb{E}\left\|\frac{1}{N} \sum_{i=1}^{N} H\left(x-z_{t_{k}}^{i}\right)-\frac{1}{N} \sum_{i=1}^{N} H\left(x-\bar{z}_{t_{k}}^{i}\right)\right\|_{L^{1}(\mathbb{R})} \\
& +\mathbb{E}\left\|\frac{1}{N} \sum_{i=1}^{N} H\left(x-\bar{z}_{t_{k}}^{i}\right)-\frac{1}{N} \sum_{i=1}^{N} H\left(x-Y_{t_{k}}^{i}\right)\right\|_{L^{1}(\mathbb{R})} .
\end{aligned}
$$

The first term on the right-hand side corresponds to the approximation error of the measure $\mu_{0}$; the second term essentially is a statistical error related to the strong Law of Large Numbers; the third term is the discretization error induced by the Euler scheme; the last term corresponds to the approximation of the coefficients 
$\beta\left(t_{k}, \cdot\right)$ and $\sigma\left(t_{k}, \cdot\right)$ by means of the empirical measure $\bar{\mu}_{t_{k}}$, which introduces the family of dependent processes $\left(Y_{t_{k}}^{i}\right)$.

The objective of the next subsections is to bound each term of the above error decomposition; we successively prove that for all $t \in(0, T]$

$$
\begin{aligned}
& \left\|\mathbb{E}_{\mu_{0}} H\left(x-z_{t}\right)-\mathbb{E}_{\bar{\mu}_{0}} H\left(x-z_{t}\right)\right\|_{L^{1}(\mathbb{R})} \leq C\left\|V_{0}-\bar{V}_{0}\right\|_{L^{1}(\mathbb{R})}, \\
& \mathbb{E}\left\|\mathbb{E}_{\bar{\mu}_{0}} H\left(x-z_{t}\right)-\frac{1}{N} \sum_{i=1}^{N} H\left(x-z_{t}^{i}\right)\right\|_{L^{1}(\mathbb{R})} \leq \frac{C}{\sqrt{N}},
\end{aligned}
$$

and for all $k \in\{0, \ldots, K\}$

$$
\begin{gathered}
\mathbb{E}\left\|\frac{1}{N} \sum_{i=1}^{N} H\left(x-z_{t_{k}}^{i}\right)-\frac{1}{N} \sum_{i=1}^{N} H\left(x-\bar{z}_{t_{k}}^{i}\right)\right\|_{L^{1}(\mathbb{R})} \leq C \sqrt{\Delta t} \\
\mathbb{E}\left\|\frac{1}{N} \sum_{i=1}^{N} H\left(x-\bar{z}_{t_{k}}^{i}\right)-\frac{1}{N} \sum_{i=1}^{N} H\left(x-Y_{t_{k}}^{i}\right)\right\|_{L^{1}(\mathbb{R})} \\
\leq C\left(\sqrt{\Delta t}+\frac{1}{\sqrt{N}}+\left\|V_{0}-\bar{V}_{0}\right\|_{L^{1}(\mathbb{R})}\right) .
\end{gathered}
$$

Obviously, this proves (2.4). Similar inequalities, explicitly proven in the next subsections, prove (2.5).

To complete the proof of Theorem 2.1, it then remains to estimate the approximation error of $V_{0}$ by $\bar{V}_{0}$.

To obtain the preceding estimates, we need well-known estimates for the density of $\mu_{t}$.

2.3.2. Exponential estimates for the density of $\mu_{t}$. The hypothesis (H2) implies that the functions $\sigma(\cdot, \cdot)$ and $\beta(\cdot, \cdot)$ are Lipschitz in $x$ and Hölder in $t$ : for all $t \in[0, T]$ and for all $(x, y) \in \mathbb{R}^{2}$ one has

$$
\begin{aligned}
& |\beta(t, x)-\beta(t, y)| \leq \int_{\mathbb{R}}|b(x, z)-b(y, z)| \mu_{t}(d z) \leq C|x-y|, \\
& |\sigma(t, x)-\sigma(t, y)| \leq \int_{\mathbb{R}}|s(x, z)-s(y, z)| \mu_{t}(d z) \leq C|x-y| ;
\end{aligned}
$$

on the other hand, $\beta(t, x)=\mathbb{E} b\left(x, X_{t}\right)$. Thus, for all $\theta, t \in[0, T]$, there holds

$$
|\beta(\theta, x)-\beta(t, x)| \leq \mathbb{E}\left|b\left(x, X_{\theta}\right)-b\left(x, X_{t}\right)\right| \leq C \mathbb{E}\left|X_{\theta}-X_{t}\right| \leq C|t-\theta|^{1 / 2} ;
$$

finally we note that (H1) and (H2) imply

$$
s_{*} \leq|\sigma(t, x)| \leq s^{*}
$$

for some $s^{*}$. All these properties of the functions $\beta$ and $\sigma$ imply (cf. pp. 139-150 in Friedman [8]) that the transition probability of $\left(z_{t}(y)\right)$ has a smooth density denoted by $p(t, y, z)$ which satisfies: for any $T$ and for all $\bar{\sigma}>s^{*}$, there exists a strictly positive constant $C$ such that for all $t \in(0, T]$ and all $y, z$,

$$
\begin{aligned}
p(t, y, z) & \leq \frac{C}{\sqrt{t}} \exp \left(-\frac{(z-y)^{2}}{2 \bar{\sigma}^{2} t}\right), \\
\left|\frac{\partial}{\partial y} p(t, y, z)\right| & \leq \frac{C}{t} \exp \left(-\frac{(z-y)^{2}}{2 \bar{\sigma}^{2} t}\right) .
\end{aligned}
$$


Since the processes $\left(X_{t}\right)$ and $\left(z_{t}\right)$ with the same initial law $\mu_{0}$ are identical in law, the law $\mu_{t}$ has a density denoted by $u(t, \cdot)$ given by

$$
u(t, z)=\int_{\mathbb{R}} p(t, y, z) \mu_{0}(d y), \forall z \in \mathbb{R}, \forall t>0 .
$$

2.3.3. Error induced by the approximation of the initial condition. Under (H3-i), we have

$$
\left\|\mathbb{E}_{\mu_{0}} H\left(x-z_{t}\right)-\mathbb{E}_{\bar{\mu}_{0}} H\left(x-z_{t}\right)\right\|_{L^{1}(\mathbb{R})}=0 .
$$

Under (H3-ii), this error is described by

Lemma 2.4. Assume (H1)-(H3-ii). There exists a positive constant $C$ depending only on $T, b$ and $\sigma$, such that, for any $t \in[0, T]$,

$$
\left\|\mathbb{E}_{\mu_{0}} H\left(x-z_{t}\right)-\mathbb{E}_{\bar{\mu}_{0}} H\left(x-z_{t}\right)\right\|_{L^{1}(\mathbb{R})} \leq C\left\|V_{0}-\bar{V}_{0}\right\|_{L^{1}(\mathbb{R})} .
$$

Furthermore,

$$
\left\|V_{0}-\bar{V}_{0}\right\|_{L^{1}(\mathbb{R})} \leq \frac{C \sqrt{\log (N)}}{N}
$$

where $C$ depends on $M, \eta$ and $\alpha$. If $\mu_{0}$ has compact support, then one even has

$$
\left\|V_{0}-\bar{V}_{0}\right\|_{L^{1}(\mathbb{R})} \leq \frac{C}{N} .
$$

Proof. We observe that

$$
\begin{aligned}
\mathbb{E}_{\bar{\mu}_{0}} H\left(x-z_{t}\right) & =\int_{\mathbb{R}} \mathbb{E} H\left(x-z_{t}(y)\right) \bar{\mu}_{0}(d y)=\int_{\mathbb{R}} \mathbb{E} H\left(x-z_{t}(y)\right) d \bar{V}_{0}(y) \\
& =\int_{-\infty}^{0} \mathbb{E} H\left(x-z_{t}(y)\right) d \bar{V}_{0}(y)-\int_{0}^{+\infty} \mathbb{E} H\left(x-z_{t}(y)\right) d\left(1-\bar{V}_{0}(y)\right) .
\end{aligned}
$$

The integration by parts formula for a Stieltjes integral gives

$$
\begin{aligned}
\mathbb{E}_{\bar{\mu}_{0}} H\left(x-z_{t}\right)= & \mathbb{E} H\left(x-z_{t}(0)\right) \bar{V}_{0}(0)-\int_{-\infty}^{0} \frac{\partial}{\partial y} \mathbb{E} H\left(x-z_{t}(y)\right) \bar{V}_{0}(y) d y \\
& +\mathbb{E} H\left(x-z_{t}(0)\right)\left(1-\bar{V}_{0}(0)\right) \\
& +\int_{0}^{+\infty} \frac{\partial}{\partial y} \mathbb{E} H\left(x-z_{t}(y)\right)\left(1-\bar{V}_{0}(y)\right) d y .
\end{aligned}
$$

A similar computation for $\mathbb{E}_{\mu_{0}} H\left(x-z_{t}\right)$ gives

$$
\mathbb{E}_{\mu_{0}} H\left(x-z_{t}\right)-\mathbb{E}_{\bar{\mu}_{0}} H\left(x-z_{t}\right)=-\int_{\mathbb{R}} \frac{\partial}{\partial y} \mathbb{E} H\left(x-z_{t}(y)\right)\left(V_{0}(y)-\bar{V}_{0}(y)\right) d y,
$$

so that

$$
\begin{aligned}
&\left\|\mathbb{E}_{\mu_{0}} H\left(x-z_{t}\right)-\mathbb{E}_{\bar{\mu}_{0}} H\left(x-z_{t}\right)\right\|_{L^{1}(\mathbb{R})} \\
& \quad \leq \int_{\mathbb{R}} \int_{\mathbb{R}}\left|\frac{\partial}{\partial y} \mathbb{E} H\left(x-z_{t}(y)\right)\right|\left|V_{0}(y)-\bar{V}_{0}(y)\right| d y d x .
\end{aligned}
$$

To complete the proof of (2.12), it remains to derive an upper bound for

$$
\int_{\mathbb{R}}\left|\frac{\partial}{\partial y} \mathbb{E} H\left(x-z_{t}(y)\right)\right| d x .
$$


We note

$$
\left|\frac{\partial}{\partial y} \mathbb{E} H\left(x-z_{t}(y)\right)\right|=\left|\frac{\partial}{\partial y} P\left(z_{t}(y)<x\right)\right| \leq \int_{-\infty}^{x}\left|\frac{\partial}{\partial y} p(t, y, \alpha)\right| d \alpha
$$

from (2.11) we deduce

$$
\left|\frac{\partial}{\partial y} \mathbb{E} H\left(x-z_{t}(y)\right)\right| \leq \frac{C}{t} \int_{-\infty}^{x} \exp \left(-\frac{(\alpha-y)^{2}}{2 \bar{\sigma}^{2} t}\right) d \alpha .
$$

Similarly, one has

$$
\begin{aligned}
\left|\frac{\partial}{\partial y} \mathbb{E} H\left(x-z_{t}(y)\right)\right| & =\left|\frac{\partial}{\partial y} P\left(z_{t}(y)>x\right)\right| \\
& \leq \frac{C}{t} \int_{x}^{+\infty} \exp \left(-\frac{(\alpha-y)^{2}}{2 \bar{\sigma}^{2} t}\right) d \alpha .
\end{aligned}
$$

Thus, from (2.14) one gets

$$
\begin{aligned}
\int_{-\infty}^{y}\left|\frac{\partial}{\partial y} \mathbb{E} H\left(x-z_{t}(y)\right)\right| d x & \leq \frac{C}{t} \int_{-\infty}^{y} \int_{-\infty}^{x} \exp \left(-\frac{(\alpha-y)^{2}}{2 \bar{\sigma}^{2} t}\right) d \alpha d x \\
& =\frac{C}{t} \int_{-\infty}^{0} \int_{-\infty}^{x} \exp \left(-\frac{\alpha^{2}}{2 \bar{\sigma}^{2} t}\right) d \alpha d x
\end{aligned}
$$

and from $(2.15)$

$$
\int_{y}^{+\infty}\left|\frac{\partial}{\partial y} \mathbb{E} H\left(x-z_{t}(y)\right)\right| d x \leq \frac{C}{t} \int_{0}^{+\infty} \int_{x}^{+\infty} \exp \left(-\frac{\alpha^{2}}{2 \bar{\sigma}^{2} t}\right) d \alpha d x .
$$

We now use the following estimates, which are easy to prove. Let $g_{\alpha}(x)$ be the density of a Gaussian law $N(0, \alpha)$, and let $G_{\alpha}(x)$ be its distribution function; then

$$
\begin{aligned}
& \text { (i) } \forall x \leq 0, G_{\alpha}(x)=\int_{-\infty}^{x} g_{\alpha}(y) d y \leq \frac{1}{2} \exp \left(-\frac{x^{2}}{2 \alpha^{2}}\right), \\
& \text { (ii) } \forall x \geq 0,\left(1-G_{\alpha}\right)(x)=\int_{x}^{+\infty} g_{\alpha}(y) d y \leq \frac{1}{2} \exp \left(-\frac{x^{2}}{2 \alpha^{2}}\right) .
\end{aligned}
$$

Thus,

$$
\int_{\mathbb{R}}\left|\frac{\partial}{\partial y} \mathbb{E} H\left(x-z_{t}(y)\right)\right| d x \leq C
$$

from which

$$
\left\|\mathbb{E}_{\mu_{0}} H\left(x-z_{t}\right)-\mathbb{E}_{\bar{\mu}_{0}} H\left(x-z_{t}\right)\right\|_{L^{1}(\mathbb{R})} \leq C \int_{\mathbb{R}}\left|V_{0}(y)-\bar{V}_{0}(y)\right| d y,
$$

which proves (2.12).

To prove (2.13), we observe

$$
\begin{aligned}
\left\|V_{0}-\bar{V}_{0}\right\|_{L^{1}(\mathbb{R})}= & \int_{-\infty}^{y_{0}^{1}} V_{0}(x) d x+\sum_{i=1}^{N-1} \int_{y_{0}^{i}}^{y_{0}^{i+1}}\left(V_{0}(x)-\frac{i}{N}\right) d x \\
& +\int_{y_{0}^{N}}^{+\infty}\left(1-V_{0}(x)\right) d x
\end{aligned}
$$


Then, using (H3-ii), we find by easy computations (see [2] for details) that

$$
\left\|V_{0}-\bar{V}_{0}\right\|_{L^{1}(\mathbb{R})} \leq \frac{C}{N}\left(1+\frac{\sqrt{\log (N)}}{N}\right)
$$

in the general case, and

$$
\left\|V_{0}-\bar{V}_{0}\right\|_{L^{1}(\mathbb{R})} \leq \frac{C}{N}
$$

in the case where $\mu_{0}$ has compact support.

2.3.4. The statistical error. The statistical error is described by

Lemma 2.5. There exists a positive constant $C$ depending on $T, b, \sigma$ and $\mu_{0}$ such that for all $t \in[0, T]$ one has

$$
\mathbb{E}\left\|\mathbb{E}_{\bar{\mu}_{0}} H\left(x-z_{t}\right)-\frac{1}{N} \sum_{i=1}^{N} H\left(x-z_{t}^{i}\right)\right\|_{L^{1}(\mathbb{R})} \leq \frac{C}{\sqrt{N}}
$$

and

$$
\mathbb{E}\left(\left\|\mathbb{E}_{\bar{\mu}_{0}} H\left(x-z_{t}\right)-\frac{1}{N} \sum_{i=1}^{N} H\left(x-z_{t}^{i}\right)\right\|_{L^{1}(\mathbb{R})}\right)^{2} \leq \frac{C}{N} .
$$

Proof. By definition of the processes $\left(z_{t}^{i}\right)$, there holds

$$
\mathbb{E}_{\bar{\mu}_{0}} H\left(x-z_{t}\right)=\frac{1}{N} \sum_{i=1}^{N} \mathbb{E} H\left(x-z_{t}\left(y_{0}^{i}\right)\right)=\frac{1}{N} \sum_{i=1}^{N} \mathbb{E} H\left(x-z_{t}^{i}\right) .
$$

Thus, the statement (i) is related to the usual Central Limit Theorem, but is not a consequence of it: we want a nonasymptotic result ( $N$ is arbitrary), and above all the random error on

$$
\mathbb{E}_{\bar{\mu}_{0}} H\left(x-z_{t}\right)
$$

owing to the Strong Law of Large Number, which depends on $x$, must be controlled in $L^{1}(\mathbb{R})$ with respect to $x$.

Set

$$
\begin{aligned}
A & :=\mathbb{E}\left\|\frac{1}{N} \sum_{i=1}^{N}\left[\mathbb{E} H\left(x-z_{t}^{i}\right)-H\left(x-z_{t}^{i}\right)\right]\right\|_{L^{1}(\mathbb{R})} \\
& \leq \int_{\mathbb{R}} \sqrt{\mathbb{E}\left|\frac{1}{N} \sum_{i=1}^{N}\left[\mathbb{E} H\left(x-z_{t}^{i}\right)-H\left(x-z_{t}^{i}\right)\right]\right|^{2}} d x .
\end{aligned}
$$

The $\left(z_{t}^{i}\right)_{i=1}^{N}$ being independent, one gets

$$
\begin{aligned}
\mathbb{E}\left|\frac{1}{N} \sum_{i=1}^{N}\left[\mathbb{E} H\left(x-z_{t}^{i}\right)-H\left(x-z_{t}^{i}\right)\right]\right|^{2} & =\frac{1}{N^{2}} \sum_{i=1}^{N} \mathbb{E}\left[\mathbb{E} H\left(x-z_{t}^{i}\right)-H\left(x-z_{t}^{i}\right)\right]^{2} \\
& =\frac{1}{N^{2}} \sum_{i=1}^{N} \mathbb{E} H\left(x-z_{t}^{i}\right) \times \mathbb{E} H\left(z_{t}^{i}-x\right),
\end{aligned}
$$


so that

$$
A \leq \frac{1}{\sqrt{N}} \int_{\mathbb{R}} \sqrt{\frac{1}{N} \sum_{i=1}^{N} \mathbb{E} H\left(x-z_{t}^{i}\right) \times \mathbb{E} H\left(z_{t}^{i}-x\right)} d x .
$$

Let $\Gamma_{t}^{i}$ denote the density of the law of $z_{t}^{i}$; one obtains

$$
A \leq \frac{1}{\sqrt{N}} \int_{\mathbb{R}} \sqrt{\frac{1}{N} \sum_{i=1}^{N} \int_{-\infty}^{x} \Gamma_{t}^{i}(y) d y \times \int_{x}^{+\infty} \Gamma_{t}^{i}(y) d y} d x .
$$

From (2.11), there exists a constant $C$ such that, for $t>0$,

$$
\Gamma_{t}^{i}(y) \leq \frac{C}{\sqrt{t}} \exp \left(-\frac{\left(y-y_{0}^{i}\right)^{2}}{2 \bar{\sigma}^{2} t}\right), \forall y \in \mathbb{R} .
$$

Thus,

$$
A \leq \frac{C}{\sqrt{N}} \int_{\mathbb{R}} \sqrt{\frac{1}{N} \sum_{i=1}^{N} \int_{-\infty}^{x-y_{0}^{i}} \frac{1}{\sqrt{t}} \exp \left(-\frac{y^{2}}{2 \bar{\sigma}^{2} t}\right) d y \times \int_{x-y_{0}^{i}}^{+\infty} \frac{1}{\sqrt{t}} \exp \left(-\frac{y^{2}}{2 \bar{\sigma}^{2} t}\right) d y} d x .
$$

In the Appendix we prove the following lemma, which completes the proof of (i):

Lemma 2.6. Under the hypothesis (H3), one has

$$
\int_{\mathbb{R}} \sqrt{\frac{1}{N} \sum_{i=1}^{N} \int_{-\infty}^{x-y_{0}^{i}} \frac{1}{\sqrt{t}} \exp \left(-\frac{y^{2}}{2 \bar{\sigma}^{2} t}\right) d y \times \int_{x-y_{0}^{i}}^{+\infty} \frac{1}{\sqrt{t}} \exp \left(-\frac{y^{2}}{2 \bar{\sigma}^{2} t}\right) d y} d x \leq C .
$$

We now prove (ii). We have

$$
\begin{aligned}
\mathbb{E}\left\|\mathbb{E}_{\bar{\mu}_{0}} H\left(x-z_{t}\right)-\frac{1}{N} \sum_{i=1}^{N} H\left(x-z_{t}^{i}\right)\right\|_{L^{1}(\mathbb{R})}^{2} \\
=\mathbb{E}\left(\int_{\mathbb{R}}\left|\frac{1}{N} \sum_{i=1}^{N} \mathbb{E} H\left(x-z_{t}^{i}\right)-H\left(x-z_{t}^{i}\right)\right| d x\right)^{2} \\
=\int_{\mathbb{R}} \int_{\mathbb{R}} \mathbb{E}\left(\left|\frac{1}{N} \sum_{i=1}^{N} \mathbb{E} H\left(x_{1}-z_{t}^{i}\right)-H\left(x_{1}-z_{t}^{i}\right)\right|\right. \\
\left.\times\left|\frac{1}{N} \sum_{i=1}^{N} \mathbb{E} H\left(x_{2}-z_{t}^{i}\right)-H\left(x_{2}-z_{t}^{i}\right)\right|\right) d x_{1} d x_{2}
\end{aligned}
$$

and we apply the Cauchy-Schwarz inequality and (i).

2.3.5. The discretization error. The aim of this subsection is to prove the following lemma. 
Lemma 2.7. For all $k=1, \ldots, K$, one has

$$
\mathbb{E}\left\|\frac{1}{N} \sum_{i=1}^{N} H\left(x-z_{t_{k}}^{i}\right)-\frac{1}{N} \sum_{i=1}^{N} H\left(x-\bar{z}_{t_{k}}^{i}\right)\right\|_{L^{1}(\mathbb{R})} \leq C \sqrt{\Delta t}
$$

and

$$
\text { (ii) } \quad \mathbb{E}\left(\left\|\frac{1}{N} \sum_{i=1}^{N} H\left(x-z_{t_{k}}^{i}\right)-\frac{1}{N} \sum_{i=1}^{N} H\left(x-\bar{z}_{t_{k}}^{i}\right)\right\|_{L^{1}(\mathbb{R})}\right)^{2} \leq C \Delta t .
$$

Proof. Noting that

$$
\forall a, b \in \mathbb{R}, \quad \int_{\mathbb{R}}|H(x-a)-H(x-b)| d x=|a-b|,
$$

one gets

$$
\mathbb{E}\left\|\frac{1}{N} \sum_{i=1}^{N} H\left(x-z_{t_{k}}^{i}\right)-\frac{1}{N} \sum_{i=1}^{N} H\left(x-\bar{z}_{t_{k}}^{i}\right)\right\|_{L^{1}(\mathbb{R})} \leq \frac{1}{N} \sum_{i=1}^{N}\left|z_{t_{k}}^{i}-\bar{z}_{t_{k}}^{i}\right|
$$

and

$$
\mathbb{E}\left(\left\|\frac{1}{N} \sum_{i=1}^{N} H\left(x-z_{t_{k}}^{i}\right)-\frac{1}{N} \sum_{i=1}^{N} H\left(x-\bar{z}_{t_{k}}^{i}\right)\right\|_{L^{1}(\mathbb{R})}\right)^{2} \leq \frac{1}{N} \sum_{i=1}^{N} \mathbb{E}\left|z_{t_{k}}^{i}-\bar{z}_{t_{k}}^{i}\right|^{2} .
$$

The quadratic mean convergence with order $\sqrt{\Delta t}$ of the Euler scheme for SDEs with coefficients which are Lipschitz functions in $x$ and Hölder of order $1 / 2$ in $t$ is an easy generalization of a result of Milshtein [22]; see M. Bossy's thesis [2] for details.

2.3.6. The dependency error. In this subsection we study the error caused by the substitution of the dependent $Y^{i}$ s to the independent $\bar{z}^{i}$ s.

Lemma 2.8. For all $k=1, \ldots, K$, there holds

$$
\begin{gathered}
\mathbb{E}\left\|\frac{1}{N} \sum_{i=1}^{N} H\left(x-\bar{z}_{t_{k}}^{i}\right)-\frac{1}{N} \sum_{i=1}^{N} H\left(x-Y_{t_{k}}^{i}\right)\right\|_{L^{1}(\mathbb{R})} \\
\leq C\left(\sqrt{\Delta t}+\frac{1}{\sqrt{N}}+\left\|V_{0}-\bar{V}_{0}\right\|_{L^{1}(\mathbb{R})}\right)
\end{gathered}
$$

and

$$
\begin{aligned}
& \mathbb{E}\left(\left\|\frac{1}{N} \sum_{i=1}^{N} H\left(x-\bar{z}_{t_{k}}^{i}\right)-\frac{1}{N} \sum_{i=1}^{N} H\left(x-Y_{t_{k}}^{i}\right)\right\|_{L^{1}(\mathbb{R})}\right)^{2} \\
& \leq C\left(\Delta t+\frac{1}{N}+\left\|V_{0}-\bar{V}_{0}\right\|_{L^{1}(\mathbb{R})}^{2}\right) .
\end{aligned}
$$

Proof. From (2.17) it follows that

$$
\left\|\frac{1}{N} \sum_{i=1}^{N} H\left(x-\bar{z}_{t_{k}}^{i}\right)-H\left(x-Y_{t_{k}}^{i}\right)\right\|_{L^{1}(\mathbb{R})} \leq \frac{1}{N} \sum_{i=1}^{N}\left|\bar{z}_{t_{k}}^{i}-Y_{t_{k}}^{i}\right| .
$$


Note that

$$
\begin{array}{r}
\mathbb{E}\left|\bar{z}_{t_{k}}^{i}-Y_{t_{k}}^{i}\right|^{2} \\
\leq \mathbb{E}\left|\bar{z}_{t_{k-1}}^{i}-Y_{t_{k-1}}^{i}\right|^{2}+\Delta t^{2} \mathbb{E}\left|\int b\left(\bar{z}_{t_{k-1}}^{i}, y\right) \mu_{t_{k-1}}(d y)-\frac{1}{N} \sum_{j=1}^{N} b\left(Y_{t_{k-1}}^{i}, Y_{t_{k-1}}^{j}\right)\right|^{2} \\
+\Delta t \mathbb{E}\left|\int s\left(\bar{z}_{t_{k-1}}^{i}, y\right) \mu_{t_{k-1}}(d y)-\frac{1}{N} \sum_{j=1}^{N} s\left(Y_{t_{k-1}}^{i}, Y_{t_{k-1}}^{j}\right)\right|^{2} \\
+2 \Delta t \mathbb{E}\left\{\left|\bar{z}_{t_{k-1}}^{i}-Y_{t_{k-1}}^{i}\right|\left|\int b\left(\bar{z}_{t_{k-1}}^{i}, y\right) \mu_{t_{k-1}}(d y)-\frac{1}{N} \sum_{j=1}^{N} b\left(Y_{t_{k-1}}^{i}, Y_{t_{k-1}}^{j}\right)\right|\right\} .
\end{array}
$$

Set $E_{k}:=\frac{1}{N} \sum_{i=1}^{N} \mathbb{E}\left|\bar{z}_{t_{k}}^{i}-Y_{t_{k}}^{i}\right|^{2}$; one has

$$
\begin{aligned}
E_{k} \leq & E_{k-1}+\Delta t^{2} \frac{1}{N} \sum_{i=1}^{N} \mathbb{E}\left|\int b\left(\bar{z}_{t_{k-1}}^{i}, y\right) \mu_{t_{k-1}}(d y)-\frac{1}{N} \sum_{j=1}^{N} b\left(Y_{t_{k-1}}^{i}, Y_{t_{k-1}}^{j}\right)\right|^{2} \\
& +\Delta t \frac{1}{N} \sum_{i=1}^{N} \mathbb{E}\left|\int s\left(\bar{z}_{t_{k-1}}^{i}, y\right) \mu_{t_{k-1}}(d y)-\frac{1}{N} \sum_{j=1}^{N} s\left(Y_{t_{k-1}}^{i}, Y_{t_{k-1}}^{j}\right)\right|^{2} \\
& +2 \Delta t \sqrt{E_{k-1}} \times \sqrt{\frac{1}{N} \sum_{i=1}^{N} \mathbb{E}\left|\int b\left(\bar{z}_{t_{k-1}}^{i}, y\right) \mu_{t_{k-1}}(d y)-\frac{1}{N} \sum_{j=1}^{N} b\left(Y_{t_{k-1}}^{i}, Y_{t_{k-1}}^{j}\right)\right|^{2}} \\
= & : E_{k-1}+A_{1}+A_{2}+A_{3} .
\end{aligned}
$$

The term $A_{1}$ is bounded from above by $C(\Delta t)^{2}$. We estimate $A_{2}$ in the following way:

$$
\begin{aligned}
\frac{1}{N} \sum_{i=1}^{N} \mathbb{E}\left|\int s\left(\bar{z}_{t_{k-1}}^{i}, y\right) \mu_{t_{k-1}}(d y)-\frac{1}{N} \sum_{j=1}^{N} s\left(Y_{t_{k-1}}^{i}, Y_{t_{k-1}}^{j}\right)\right|^{2} \\
\leq \frac{2}{N} \sum_{i=1}^{N} \mathbb{E}\left|\int s\left(\bar{z}_{t_{k-1}}^{i}, y\right) \mu_{t_{k-1}}(d y)-\frac{1}{N} \sum_{j=1}^{N} s\left(\bar{z}_{t_{k-1}}^{i}, \bar{z}_{t_{k-1}}^{j}\right)\right|^{2} \\
\quad+\frac{2}{N} \sum_{i=1}^{N} \mathbb{E}\left|\frac{1}{N} \sum_{j=1}^{N} s\left(\bar{z}_{t_{k-1}}^{i}, \bar{z}_{t_{k-1}}^{j}\right)-s\left(Y_{t_{k-1}}^{i}, Y_{t_{k-1}}^{j}\right)\right|^{2}
\end{aligned}
$$

As the function $s$ is Lipschitz, we observe

$$
\frac{2}{N} \sum_{i=1}^{N} \mathbb{E}\left|\frac{1}{N} \sum_{j=1}^{N} s\left(\bar{z}_{t_{k-1}}^{i}, \bar{z}_{t_{k-1}}^{j}\right)-s\left(Y_{t_{k-1}}^{i}, Y_{t_{k-1}}^{j}\right)\right|^{2} \leq C E_{k-1}
$$


Now set $\tilde{\mu}_{t_{k}}:=\frac{1}{N} \sum_{j=1}^{N} \delta_{\bar{z}_{t_{k}}^{j}}$ and $\widetilde{V}_{t_{k}}(x):=\frac{1}{N} \sum_{j=1}^{N} H\left(x-\bar{z}_{t_{k}}^{j}\right)$. Note that

$$
\begin{gathered}
\int s\left(\bar{z}_{t_{k-1}}^{i}, y\right) \mu_{t_{k-1}}(d y)-\frac{1}{N} \sum_{j=1}^{N} s\left(\bar{z}_{t_{k-1}}^{i}, \bar{z}_{t_{k-1}}^{j}\right) \\
=\int s\left(\bar{z}_{t_{k-1}}^{i}, y\right)\left(\mu_{t_{k-1}}(d y)-\tilde{\mu}_{t_{k-1}}(d y)\right) ;
\end{gathered}
$$

as the function $s$ is differentiable, one obtains

$$
\begin{aligned}
\int s\left(\bar{z}_{t_{k-1}}^{i}, y\right)\left(\mu_{t_{k-1}}(d y)-\tilde{\mu}_{t_{k-1}}(d y)\right) & =\int \frac{\partial s}{\partial y}\left(\bar{z}_{t_{k-1}}^{i}, y\right)\left(V\left(t_{k-1}, y\right)-\widetilde{V}_{t_{k-1}}(y)\right) d y \\
& \leq C\left\|V\left(t_{k-1}, x\right)-\widetilde{V}_{t_{k-1}}(x)\right\|_{L^{1}(\mathbb{R})} .
\end{aligned}
$$

From (2.9) and Lemmas 2.4, 2.5 and 2.7, it follows that

$$
\mathbb{E}\left(\left\|V\left(t_{k-1}, x\right)-\widetilde{V}_{t_{k-1}}(x)\right\|_{L^{1}(\mathbb{R})}\right)^{2} \leq C\left(\left\|V_{0}-\bar{V}_{0}\right\|_{L^{1}(\mathbb{R})}^{2}+\frac{1}{N}+\Delta t\right),
$$

from which

$$
A_{2} \leq C \Delta t\left(\left\|V_{0}-\bar{V}_{0}\right\|_{L^{1}(\mathbb{R})}^{2}+\frac{1}{N}+\Delta t\right)+C \Delta t E_{k-1} .
$$

Now consider $A_{3}$. We need a precise estimate on $\sqrt{A_{1}}$.

As $\mu_{t_{k-1}}$ is the law of $z_{t_{k-1}}$, one has that $\int b(x, y) \mu_{t_{k-1}}(d y)=\mathbb{E}_{\mu_{0}} b\left(x, z_{t_{k-1}}\right)$. We set

$$
\left.\mathbb{E}_{\mu_{0}} b\left(x, z_{t_{k-1}}\right)\right|_{{\overline{t_{k-1}}}_{t_{k-1}}}:=\left.\mathbb{E}_{\mu_{0}} b\left(x, z_{t_{k-1}}\right)\right|_{x=\bar{z}_{t_{k-1}}^{i}}:=\int b\left(\bar{z}_{t_{k-1}}^{i}, y\right) \mu_{t_{k-1}}(d y) .
$$

Observe that

$$
\begin{aligned}
& \frac{1}{N} \sum_{i=1}^{N} \mathbb{E}\left|\int b\left(\bar{z}_{t_{k-1}}^{i}, y\right) \mu_{t_{k-1}}(d y)-\frac{1}{N} \sum_{j=1}^{N} b\left(Y_{t_{k-1}}^{i}, Y_{t_{k-1}}^{j}\right)\right|^{2} \\
& \leq \frac{2}{N} \sum_{i=1}^{N} \mathbb{E}\left|\mathbb{E}_{\mu_{0}} b\left(x, z_{t_{k-1}}\right)\right|_{\bar{z}_{t_{k-1}}^{i}}-\left.\mathbb{E}_{\bar{\mu}_{0}} b\left(x, z_{t_{k-1}}\right)\right|_{\bar{z}_{t_{k-1}}^{i}} \mid \\
&+ \frac{2}{N} \sum_{i=1}^{N} \mathbb{E}\left|\mathbb{E}_{\bar{\mu}_{0}} b\left(x, z_{t_{k-1}}\right)\right|_{\bar{z}_{t_{k-1}}^{i}}-\left.\left.\frac{1}{N} \sum_{j=1}^{N} \mathbb{E} b\left(x, \bar{z}_{t_{k-1}}^{j}\right)\right|_{\bar{z}_{t_{k-1}}^{i}}\right|^{2} \\
&+\frac{2}{N} \sum_{i=1}^{N} \mathbb{E}\left|\frac{1}{N} \sum_{j=1}^{N} \mathbb{E} b\left(x, \bar{z}_{t_{k-1}}^{j}\right)\right|_{\bar{z}_{t_{k-1}}^{i}}-\left.\frac{1}{N} \sum_{j=1}^{N} b\left(\bar{z}_{t_{k-1}}^{i}, \bar{z}_{t_{k-1}}^{j}\right)\right|^{2} \\
&+\frac{2}{N} \sum_{i=1}^{N} \mathbb{E}\left|\frac{1}{N} \sum_{j=1}^{N} b\left(\bar{z}_{t_{k-1}}^{i}, \bar{z}_{t_{k-1}}^{j}\right)-b\left(Y_{t_{k-1}}^{i}, Y_{t_{k-1}}^{j}\right)\right|^{2} \\
&=: \varepsilon_{k-1}^{1}+\varepsilon_{k-1}^{2}+\varepsilon_{k-1}^{3}+\varepsilon_{k-1}^{4} .
\end{aligned}
$$


A preceding computation shows

$$
\varepsilon_{k-1}^{4} \leq C E_{k-1}
$$

On the other hand, one has

$$
\begin{aligned}
\varepsilon_{k-1}^{3}= & \frac{2}{N^{3}} \sum_{i=1}^{N} \sum_{j=1}^{N} \mathbb{E}\left(\left.\mathbb{E} b\left(x, \bar{z}_{t_{k-1}}^{j}\right)\right|_{\bar{z}_{t_{k-1}}^{i}}-b\left(\bar{z}_{t_{k-1}}^{i}, \bar{z}_{t_{k-1}}^{j}\right)\right)^{2} \\
+\frac{2}{N^{3}} \sum_{i=1}^{N} \sum_{(j, l=1 ; j \neq l)}^{N} \mathbb{E}\{ & {\left[\left.\mathbb{E} b\left(x, \bar{z}_{t_{k-1}}^{j}\right)\right|_{\bar{z}_{t_{k-1}}^{i}}-b\left(\bar{z}_{t_{k-1}}^{i}, \bar{z}_{t_{k-1}}^{j}\right)\right] } \\
& \left.\times\left[\left.\mathbb{E} b\left(x, \bar{z}_{t_{k-1}}^{l}\right)\right|_{z_{t_{k-1}}^{i}}-b\left(\bar{z}_{t_{k-1}}^{i}, \bar{z}_{t_{k-1}}^{l}\right)\right]\right\} .
\end{aligned}
$$

Since the $\bar{z}^{i}$ s are independent and $b$ is bounded, one deduces

$$
\varepsilon_{k-1}^{3} \leq \frac{C}{N}
$$

Now observe that

$$
\begin{aligned}
\varepsilon_{k-1}^{2} & =\frac{2}{N} \sum_{i=1}^{N} \mathbb{E}\left|\frac{1}{N} \sum_{j=1}^{N} \mathbb{E} b\left(x, z_{t_{k-1}}\left(y_{0}^{j}\right)\right)\right|_{\bar{z}_{t_{k-1}}^{i}}-\left.\left.\frac{1}{N} \sum_{j=1}^{N} \mathbb{E} b\left(x, \bar{z}_{t_{k-1}}^{j}\right)\right|_{\bar{z}_{t_{k-1}}^{i}}\right|^{2} \\
& =\left.\frac{2}{N} \sum_{i=1}^{N} \mathbb{E}\left|\frac{1}{N} \sum_{j=1}^{N} \mathbb{E}\left(b\left(x, z_{t_{k-1}}^{j}\right)-b\left(x, \bar{z}_{t_{k-1}}^{j}\right)\right)\right|_{\bar{z}_{t_{k-1}}^{i}}\right|^{2},
\end{aligned}
$$

from which

$$
\varepsilon_{k-1}^{2} \leq C\left\{\frac{1}{N} \sum_{j=1}^{N} \mathbb{E}\left|z_{t_{k-1}}^{j}-\bar{z}_{t_{k-1}}^{j}\right|\right\}^{2} .
$$

Applying Lemma 2.7, we conclude that

$$
\varepsilon_{k-1}^{2} \leq C \Delta t
$$

It remains to estimate $\varepsilon_{k-1}^{1}$. Note that

$$
\begin{aligned}
\left.\mathbb{E}_{\mu_{0}} b\left(x, z_{t_{k-1}}\right)\right|_{\bar{z}_{t_{k-1}}^{i}}-\left.\mathbb{E}_{\bar{\mu}_{0}} b\left(x, z_{t_{k-1}}\right)\right|_{\bar{z}_{t_{k-1}}^{i}} \\
=\left.\left(\mathbb{E}_{\mu_{0}} b\left(x, z_{t_{k-1}}\right)-\mathbb{E}_{\bar{\mu}_{0}} b\left(x, z_{t_{k-1}}\right)\right)\right|_{\bar{z}_{t_{k-1}}^{i}} ;
\end{aligned}
$$

moreover, for all $x$,

$$
\mathbb{E}_{\mu_{0}} b\left(x, z_{t_{k-1}}\right)-\mathbb{E}_{\bar{\mu}_{0}} b\left(x, z_{t_{k-1}}\right)=\int_{\mathbb{R}} \mathbb{E} b\left(x, z_{t_{k-1}}(y)\right)\left(\mu_{0}(d y)-\bar{\mu}_{0}(d y)\right) .
$$

Now integrate by parts and apply (2.11); it then follows that, for all $x$,

$$
\left|\mathbb{E}_{\mu_{0}} b\left(x, z_{t_{k-1}}\right)-\mathbb{E}_{\bar{\mu}_{0}} b\left(x, z_{t_{k-1}}\right)\right| \leq \frac{C}{\sqrt{t_{k-1}}}\left\|V_{0}-\bar{V}_{0}\right\|_{L^{1}(\mathbb{R})},
$$


so that, for $k>1$,

$$
\varepsilon_{k-1}^{1} \leq \frac{C}{t_{k-1}}\left\|V_{0}-\bar{V}_{0}\right\|_{L^{1}(\mathbb{R})}^{2} .
$$

Combining (2.20), (2.21), (2.22), (2.23), one gets

$$
A_{3} \leq C \Delta t \sqrt{E_{k-1}} \cdot \sqrt{\frac{\left\|V_{0}-\bar{V}_{0}\right\|_{L^{1}(\mathbb{R})}^{2}}{t_{k-1}}+\Delta t+\frac{1}{N}}+C \Delta t E_{k-1} .
$$

Set

$$
\delta:=\left\|V_{0}-\bar{V}_{0}\right\|_{L^{1}(\mathbb{R})}^{2}+\frac{1}{N}+\Delta t .
$$

In view of this upper bound and (2.19), the inequality (2.18) becomes

$$
\left\{\begin{array}{l}
E_{k} \leq(1+C \Delta t) E_{k-1}+C \Delta t(\delta+\Delta t)+C \Delta t \frac{\sqrt{E_{k-1}}}{\sqrt{t_{k-1}}} \sqrt{\delta} \text { for } k>1, \\
E_{1} \leq C \Delta t .
\end{array}\right.
$$

Consider the sequence $\left(\gamma_{k}\right)$ defined by

$$
\left\{\begin{array}{l}
\gamma_{k}:=(1+C \Delta t) \gamma_{k-1}+C \Delta t(\delta+\Delta t)+C \Delta t \frac{\sqrt{\gamma_{k-1}}}{\sqrt{t_{k-1}}} \sqrt{\delta} \text { for } k>1, \\
\gamma_{1}:=C \Delta t .
\end{array}\right.
$$

Then, for all $k=1, \ldots, K$, we have $E_{k} \leq \gamma_{k}$. Suppose that there exists an integer $q<K$ such that

$$
\gamma_{q} \leq \delta \text { and } \gamma_{q+1} \geq \delta .
$$

As $\left(\gamma_{k}\right)$ is increasing, one then has

$$
\begin{array}{ll}
\forall r \leq q, & \gamma_{r} \leq\left\|V_{0}-\bar{V}_{0}\right\|_{L^{1}(\mathbb{R})}^{2}+\frac{1}{N}+\Delta t, \\
\forall r \geq q+1, & \gamma_{r} \geq\left\|V_{0}-\bar{V}_{0}\right\|_{L^{1}(\mathbb{R})}^{2}+\frac{1}{N}+\Delta t .
\end{array}
$$

Thus,

$$
\left\{\begin{array}{l}
\gamma_{k} \leq\left(1+C \Delta t+C \frac{\Delta t}{\sqrt{t_{k-1}}}\right) \gamma_{k-1}+C \Delta t(\delta+\Delta t), k=q+2, \ldots, K, \\
\gamma_{q+1} \leq C \delta .
\end{array}\right.
$$

Noting that $\sum_{j=q}^{K-1} \frac{1}{\sqrt{j}} \leq \int_{q}^{K} \frac{1}{\sqrt{x}} d x=2(\sqrt{K}-\sqrt{q})$, an induction gives

$$
\gamma_{K} \leq C \delta
$$

The conclusion follows from the definition of $\delta$ (see (2.24)). 
2.4. Proof of Theorem 2.2. Under the hypothesis (H2'), the functions $\beta$ and $\sigma$ are of class $\mathcal{C}^{2}(\mathbb{R})$; then $(\mathrm{H} 1)$ and standard arguments imply that $u(t, \cdot)$, the density of the law of $z_{t}$ solution to (2.6), is a classical solution to the PDE (1.3).

We observe that

$$
\begin{aligned}
\left\|u\left(t_{k}, \cdot\right)-\bar{u}_{t_{k}}^{\varepsilon}(\cdot)\right\|_{L^{1}(\mathbb{R})} \leq & \left\|u\left(t_{k}, \cdot\right)-\left(u\left(t_{k}, \cdot\right) * \Phi_{\varepsilon}\right)\right\|_{L^{1}(\mathbb{R})} \\
& +\left\|\left(u\left(t_{k}, \cdot\right)-\bar{u}_{t_{k}}(\cdot)\right) * \Phi_{\varepsilon}\right\|_{L^{1}(\mathbb{R})} .
\end{aligned}
$$

The first term of the right-hand side corresponds to the rate of convergence of the regularization by a Gaussian kernel $\Phi_{\varepsilon}$. Below we show that the density $u(t, \cdot)$ belongs to the Sobolev space $W^{2,1}(\mathbb{R})$. Thus, we can use the well-known estimate (cf. Raviart [26])

$$
\left\|u\left(t_{k}, \cdot\right)-\left(u\left(t_{k}, \cdot\right) * \Phi_{\varepsilon}\right)\right\|_{L^{1}(\mathbb{R})} \leq C \varepsilon^{2}\left\|u\left(t_{k}, \cdot\right)\right\|_{W^{2,1}(\mathbb{R})} .
$$

Using the integration by parts formula for a Stieltjes integral, the second term of (2.25) can be rewritten

$$
\left\|\left(u\left(t_{k}, \cdot\right)-\bar{u}_{t_{k}}(\cdot)\right) * \Phi_{\varepsilon}\right\|_{L^{1}(\mathbb{R})}=\int_{\mathbb{R}}\left|\int_{\mathbb{R}} \Phi_{\varepsilon}^{\prime}(x-y)\left(V\left(t_{k}, y\right)-\bar{V}_{t_{k}}(y)\right) d y\right| d x,
$$

so that

$$
\mathbb{E}\left\|\left(u\left(t_{k}, \cdot\right)-\bar{u}_{t_{k}}(\cdot)\right) * \Phi_{\varepsilon}\right\|_{L^{1}(\mathbb{R})} \leq \frac{2}{\sqrt{2 \pi} \varepsilon} \mathbb{E}\left\|V\left(t_{k}, .\right)-\bar{V}_{t_{k}}(.)\right\|_{L^{1}(\mathbb{R})} .
$$

The estimates of Theorem 2.2 are obtained by combining (2.26) and (2.27); it remains to show that the assumptions $\left(\mathrm{H} 2^{\prime}\right)$ and $\left(\mathrm{H} 3^{\prime}\right)$ ensure that the norm of $u(t, \cdot)$ in $W^{2,1}(\mathbb{R})$ is bounded uniformly in $t \in[0, T]$.

To prove this, we use a criterion due to Cannarsa and Vespri (see below) ensuring that the solution of a parabolic PDE belongs to $C^{1}\left([0, t] ; L^{2}(\mathbb{R})\right) \cap C\left([0, T] ; W^{2,2}(\mathbb{R})\right)$. Thus, we will consider a function $\tilde{u}(t, x)$, a solution of a parabolic PDE satisfying the conditions imposed by Cannarsa and Vespri, and such that, if the $W^{2,2}(\mathbb{R})$ norm of $\tilde{u}(t, \cdot)$ is uniformly bounded on $[0, T]$, then the $W^{2,1}(\mathbb{R})$ norm of $u(t, \cdot)$ is uniformly bounded on $[0, T]$. The latter is easy to prove for

$$
\tilde{u}(t, x):=\left(1+x^{2}\right) u(t, x) .
$$

We now observe that $\tilde{u}(t, x)$ solves the linear parabolic equation

$$
\left\{\begin{array}{l}
\frac{\partial \tilde{u}}{\partial t}(t, x)=L(t) \tilde{u}(t, x),(t, x) \in[0, T] \times \mathbb{R}, \\
\tilde{u}(0, x)=\exp (\pi(x)) u_{0}(x)
\end{array}\right.
$$

where

$$
\begin{gathered}
\pi(x)=\ln \left(1+x^{2}\right), \\
L(t)=\tilde{a}(t, x) \frac{\partial^{2}}{\partial x^{2}}+\tilde{b}(t, x) \frac{\partial}{\partial x}-\tilde{c}(t, x),
\end{gathered}
$$

and

$$
\begin{aligned}
& \tilde{a}:=\frac{1}{2} \sigma^{2}, \tilde{b}:=\frac{\partial\left(\sigma^{2}\right)}{\partial x}-\beta-\sigma^{2} \pi^{\prime} \\
& \tilde{c}:=\frac{\partial \beta}{\partial x}-\frac{1}{2} \frac{\partial^{2}\left(\sigma^{2}\right)}{\partial x^{2}}+\pi^{\prime}\left(\frac{\partial\left(\sigma^{2}\right)}{\partial x}-\beta\right)+\frac{1}{2} \sigma^{2}\left(\pi^{\prime \prime}-\left(\pi^{\prime}\right)^{2}\right) .
\end{aligned}
$$


We now check the different conditions required to apply the result of Cannarsa and Vespri.

As $\pi^{\prime}$ and $\pi^{\prime \prime}$ are bounded functions, under the assumption $\left(\mathrm{H} 2^{\prime}\right), \partial_{x^{p}}^{p} \sigma$ and $\partial_{x^{q}}^{q} \beta$ are bounded functions in $\mathbb{R}$ and their norms in $L^{\infty}(\mathbb{R})$ are uniform with respect to $t \in[0, T]$, for $p=0,1,2$ and $q=0,1$. Moreover, as $\beta(t, x)=\mathbb{E} b\left(x, X_{t}\right)$ and $\sigma(t, x)=$ $\mathbb{E} s\left(x, X_{t}\right)$, these functions are Hölder in $[0, T]$, uniformly on $\mathbb{R}$, with exponent $\frac{1}{2}$, (see $\S 2.3 .2)$. Finally, under $\left(\mathrm{H} 3^{\prime}\right), \tilde{u}_{0}(\cdot)$ is in $W^{2,2}(\mathbb{R})$. Therefore (see Cannarsa and Vespri [4]), the problem $(2.28)$ has a unique solution in $C^{1}\left([0, t] ; L^{2}(\mathbb{R})\right) \cap$ $C\left([0, T] ; W^{2,2}(\mathbb{R})\right)$. Thus, $x \rightarrow \exp (\pi(x)) u(t, x)$ has a $W^{2,2}(\mathbb{R})$ norm uniformly bounded with respect to $t \in[0, T]$; as already noticed, one can easily deduce that $u(t, \cdot)$ is in $W^{2,1}(\mathbb{R})$ with a norm uniformly bounded with respect to $t \in[0, T]$.

\section{The Algorithm for the Burgers EQuation With NONMONotonic INITIAL CONDITIONS}

3.1. Preliminaries. In Bossy and Talay [3] we analyze the convergence rate of the preceding stochastic particle method when the kernel $b(x, y)$ is equal to $H(x-y)$, $H$ being the Heaviside function $(H(z)=1$ if $z \geq 0, H(z)=0$ if $z<0)$, and the kernel $s(x, y)$ is the constant function $\sigma$; in view of $(1.2), V(t, x)$ solves the Burgers equation with an initial condition equal to the distribution function of a probability law:

$$
\left\{\begin{array}{l}
\frac{\partial V}{\partial t}=\frac{1}{2} \sigma^{2} \frac{\partial^{2} V}{\partial x^{2}}-V \frac{\partial V}{\partial x} \text { in }[0, T] \times \mathbb{R}, \\
V(0, x)=V_{0}(x) .
\end{array}\right.
$$

The error analysis is based upon arguments similar to those of the preceding section, plus specific and tricky computations owing to the discontinuity of $b$.

In this section, we extend the algorithm to a larger class of initial conditions by considering $V_{0}$ as the "distribution function" of a signed and finite measure. For this extension, the convergence rates given in Theorem 2.1 still hold (see $\S 3.4$ ).

Let $\mathcal{M}(\mathbb{R})$ be the set of measures on $\mathbb{R}$ and $\mathcal{M}^{+}(\mathbb{R})$ the set of positive parts of measures on $\mathcal{M}(\mathbb{R})$. For any measure $\mu$ in $\mathcal{M}(\mathbb{R})$, we denote by $\mu^{+}$and $\mu^{-}$the positive measures coming from the Jordan decomposition of $\mu$. Let $\mathcal{M}\left(\mathbb{R} ; a^{+} ; a^{-}\right)$ be defined by

$$
\mathcal{M}\left(\mathbb{R} ; a^{+} ; a^{-}\right)=\left\{\mu \in \mathcal{M}(\mathbb{R}),\left\|\mu^{+}\right\|=a^{+},\left\|\mu^{-}\right\|=a^{-}\right\},
$$

where $\|\mu\|$ denotes the total variation of $\mu$.

Suppose that $V_{0}$ satisfies

$$
V_{0}(x)=\int_{-\infty}^{x} W_{0}(d y) \text { with } W_{0} \in \mathcal{M}\left(\mathbb{R} ; a^{+} ; a^{-}\right) .
$$

Our first objective is to show that $V(t, x)=\int_{\infty}^{x} W_{t}(d y)$, where the measure $W_{t}$ belongs to $\mathcal{M}\left(\mathbb{R} ; a^{+} ; a^{-}\right)$for all $t$, and is the unique weak solution to the PDE

$$
\left\{\begin{array}{l}
\frac{\partial W_{t}}{\partial t}=\frac{1}{2} \sigma^{2} \frac{\partial^{2} W_{t}}{\partial x^{2}}-\frac{\partial}{\partial x}\left(W_{t}\left(\int_{\mathbb{R}} H(x-y) W_{t}(d y)\right)\right), \\
W_{t=0}=W_{0} .
\end{array}\right.
$$


This is done by adapting ideas of Marchioro and Pulvirenti [19] for the probabilistic interpretation of the incompressible 2-D Navier-Stokes equation.

3.2. Probabilistic interpretation. The measure $W_{0} \in \mathcal{M}\left(\mathbb{R} ; a^{+} ; a^{-}\right)$being fixed, set

$$
U_{0}^{+}:=\frac{W_{0}^{+}}{a^{+}} \quad \text { and } \quad U_{0}^{-}:=\frac{W_{0}^{-}}{a^{-}},
$$

where $W_{0}^{+}$and $W_{0}^{-}$are the positive measures coming from the Jordan decomposition of $W_{0}$. Note that $U_{0}^{+}$and $U_{0}^{-}$are probability measures. Throughout the sequel, we assume:

(H0) $U_{0}^{+}$and $U_{0}^{-}$satisfy one of the two conditions:

(i) $U_{0}^{ \pm}$has a continuous and strictly positive density $u_{0}^{ \pm}$and there exist strictly positive constants $M, \eta$ and $\alpha$ such that

$$
u_{0}^{ \pm}(x) \leq \eta \exp \left(-\alpha \frac{x^{2}}{2}\right) \text { for }|x|>M .
$$

(ii) $U_{0}^{ \pm}$has compact support included in an interval $(-M, M)$.

Theorem 3.1. Suppose that $W_{0} \in \mathcal{M}\left(\mathbb{R} ; a^{+} ; a^{-}\right)$satisfies the hypothesis (H0). Then:

(a) There exists a unique function $[0, T] \ni t \rightarrow W_{t} \in \mathcal{M}\left(\mathbb{R} ; a^{+} ; a^{-}\right)$such that, for any function $f$ in $C_{K}^{2}(\mathbb{R})$,

$$
\left\{\begin{array}{l}
\frac{d}{d t}\left(\int_{\mathbb{R}} f(y) W_{t}(d y)\right)=\frac{1}{2} \sigma^{2} \int_{\mathbb{R}} f^{\prime \prime}(y) W_{t}(d y)+\int_{\mathbb{R}} V(t, y) f^{\prime}(y) W_{t}(d y) \\
W_{t=0}=W_{0}
\end{array}\right.
$$

where $V(t, x)=\int_{\mathbb{R}} H(x-y) W_{t}(d y)$, and moreover,

$$
W_{t}(\mathcal{A})=\int_{\mathbb{R}} P_{t}\left(x_{0}, \mathcal{A}\right) W_{0}\left(d x_{0}\right),
$$

for any Borel set $\mathcal{A} \subset \mathbb{R}$, where $P_{t}\left(x_{0}, \cdot\right)$ is the transition probability of the process $\left(z_{t}\right)$ solution to

$$
d z_{t}=V\left(t, z_{t}\right) d t+\sigma d w_{t}
$$

(b) The function $V$ so defined is the classical solution to the Burgers equation in $[0, T] \times \mathbb{R}$, and for all $t \in(0, T]$ one has

$$
\left\|\frac{\partial V}{\partial x}(t, \cdot)\right\|_{L^{\infty}(\mathbb{R})} \leq \frac{C}{\sqrt{t}}
$$

where $C$ depends on $\sigma, T$ and $W_{0}$.

To prove Theorem 3.1, we need an analogous statement for a regularized problem that we now present.

For $\varepsilon>0$, denote again by $\Phi_{\varepsilon}$ the density of a Gaussian random variable of mean 0 and variance $\varepsilon^{2}$; define

$$
H^{\varepsilon}(x)=\left(H * \Phi_{\varepsilon}\right)(x)=\int_{-\infty}^{x} \frac{1}{\sqrt{2 \pi \varepsilon^{2}}} \exp \left(-\frac{y^{2}}{2 \varepsilon^{2}}\right) d y
$$


Theorem 3.2. Let $W_{0} \in \mathcal{M}\left(\mathbb{R} ; a^{+} ; a^{-}\right)$. There exists a unique function $[0, T] \ni$ $t \rightarrow W_{t}^{\varepsilon} \in \mathcal{M}\left(\mathbb{R} ; a^{+} ; a^{-}\right)$such that, for all functions $f$ in $C_{K}^{2}(\mathbb{R})$,

$$
\left\{\begin{array}{l}
\frac{d}{d t}\left(\int_{\mathbb{R}} f(y) W_{t}^{\varepsilon}(d y)\right)=\frac{1}{2} \sigma^{2} \int_{\mathbb{R}} f^{\prime \prime}(y) W_{t}^{\varepsilon}(d y)+\int_{\mathbb{R}} V^{\varepsilon}(t, y) f^{\prime}(y) W_{t}^{\varepsilon}(d y), \\
W_{t=0}=W_{0},
\end{array}\right.
$$

where $V^{\varepsilon}(t, x)=\int_{\mathbb{R}} H^{\varepsilon}(x-y) W_{t}^{\varepsilon}(d y)$, and moreover,

$$
W_{t}^{\varepsilon}(\mathcal{A})=\int_{\mathbb{R}} P_{t}^{\varepsilon}\left(x_{0}, \mathcal{A}\right) W_{0}\left(d x_{0}\right),
$$

for all Borel sets $\mathcal{A} \subset \mathbb{R}$, where $P_{t}^{\varepsilon}\left(x_{0}, \cdot\right)$ is the transition probability of the process $\left(z_{t}^{\varepsilon}\right)$ solution to

$$
d z_{t}^{\varepsilon}=V^{\varepsilon}\left(t, z_{t}\right) d t+\sigma d w_{t} .
$$

In the Appendix we give a sketch of the proof of Theorem 3.2, which adapts arguments appearing in [19].

3.3. Proof of Theorem 3.1. In [19], Marchioro and Pulvirenti assume that the initial condition of the 2-D Navier-Stokes equation is in $L^{1}(\mathbb{R}) \cap L^{\infty}(\mathbb{R})$; they use this hypothesis to go from a regularized problem (similar to (3.5)) to the NavierStokes equation. We have not succeeded in adapting their technique for our case because $W_{0}$ may be singular.

Thus, we proceed differently: first, we construct a solution to (3.3) by considering a convergent subsequence of $\left(W_{t}^{\varepsilon}\right)_{\varepsilon}$; second, we show that this solution is related to the law of the appropriate stochastic process.

Observe that for all $(t, x)$ in $[0, T] \times \mathbb{R}$, we have

$$
\left|V^{\varepsilon}(t, x)\right| \leq\left|\int_{\mathbb{R}} W_{t}^{\varepsilon}(d y)\right| \leq a^{+}+a^{-}:=A .
$$

Thus (see [3] for a proof), for all $x_{0}$ in $\mathbb{R}$ the process $\left(z_{t}^{\varepsilon}\left(x_{0}\right)\right)$ has a density, denoted by $p_{t}^{\varepsilon}\left(\cdot, x_{0}\right)$, such that

$$
\exists C>0, \forall \varepsilon>0,\left\|p_{t}^{\varepsilon}\left(\cdot, x_{0}\right)\right\|_{L^{2}(\mathbb{R})} \leq \frac{C}{t^{\frac{1}{4}}} .
$$

Denote by $\mathcal{M}^{1}(C([0, T] ; \mathbb{R}))$ the set of probability measures on $C([0, T] ; \mathbb{R})$ and consider the sequence $\left(P^{\varepsilon}\left(x_{0}\right)\right)_{\varepsilon}$ of measures belonging to $\mathcal{M}^{1}(C([0, T] ; \mathbb{R}))$ defined by

$$
P^{\varepsilon}\left(x_{0}\right)=\mathbb{P} \circ\left(z^{\varepsilon}\left(x_{0}\right)\right)^{-1} .
$$

For all $0 \leq s \leq t \leq T$, one has that

$$
\mathbb{E}\left|z_{t}^{\varepsilon}\left(x_{0}\right)-z_{s}^{\varepsilon}\left(x_{0}\right)\right| \leq \mathbb{E}\left|\sigma\left(w_{t}-w_{s}\right)\right|^{4}+A^{4}(t-s)^{4} \leq C(t-s)^{2},
$$

which implies that $\left(P^{\varepsilon}\left(x_{0}\right)\right)_{\varepsilon}$ is tight (cf. Karatzas and Shreve [15] e.g.).

Let $\widetilde{P}\left(x_{0}\right)$ be the limit of a convergent subsequence (again denoted by $\left.\left(P^{\varepsilon}\left(x_{0}\right)\right)_{\varepsilon}\right)$.

\section{Lemma 3.3.}

(i) For any $x_{0}$ in $\mathbb{R}$ and $t \in(0, T]$ the one-dimensional distribution $\widetilde{P}_{t}\left(x_{0}\right)$ of $\widetilde{P}\left(x_{0}\right)$ has a density $\widetilde{p}_{t}\left(x_{0}, \cdot\right)$ with respect to Lebesgue measure and

$$
\forall t \in(0, T], \quad\left\|\widetilde{p}_{t}\left(\cdot, x_{0}\right)\right\|_{L^{2}(\mathbb{R})} \leq \frac{C}{t^{\frac{1}{4}}} .
$$


(ii) The function $[0, T] \ni t \rightarrow \widetilde{W}_{t} \in \mathcal{M}\left(\mathbb{R} ; a^{+} ; a^{-}\right)$defined by

$$
\left\{\begin{array}{l}
\widetilde{W}_{t}(\mathcal{A})=\int_{\mathbb{R}} \widetilde{P}_{t}(x, \mathcal{A}) W_{0}(d x) \text { for all Borel sets } \mathcal{A} \subset \mathbb{R} \text { and } t \in(0, T] \\
\widetilde{W}_{0}=W_{0}
\end{array}\right.
$$

is a solution to $(3.3)$.

Proof. We first prove (i). For any $t>0$ and any function $f$ in $L^{2}(\mathbb{R})$, we have

$$
\left|\int_{\mathbb{R}} f(x) P_{t}^{\varepsilon}\left(x_{0}, d x\right)\right|=\left|\int_{\mathbb{R}} f(x) p_{t}^{\varepsilon}\left(x_{0}, x\right) d x\right| \leq \frac{C}{t^{\frac{1}{4}}}\|f\|_{L^{2}(\mathbb{R})} .
$$

From the weak convergence of $P_{t}^{\varepsilon}\left(x_{0}\right)$ to $\widetilde{P}_{t}\left(x_{0}\right)$ and (3.6) it follows that for any function $f$ in $C_{K}(\mathbb{R})$,

$$
\left|\int_{\mathbb{R}} f(x) \widetilde{P}_{t}\left(x_{0}\right)(d x)\right| \leq \frac{C}{t^{\frac{1}{4}}}\|f\|_{L^{2}(\mathbb{R})} .
$$

This implies that $\widetilde{P}_{t}\left(x_{0}, \cdot\right)$ has a density $\widetilde{p}_{t}\left(x_{0}, \cdot\right)$ in $L^{2}(\mathbb{R})$ for all $t>0$, and

$$
\left\|\widetilde{p}_{t}\left(x_{0}, \cdot\right)\right\|_{L^{2}(\mathbb{R})} \leq \frac{C}{t^{\frac{1}{4}}} .
$$

We now show (ii). We prove that for all functions $f$ in $C_{K}^{2}(\mathbb{R})$,

$$
\begin{array}{rl}
\int_{\mathbb{R}} & f(y) \widetilde{W}_{t}(d y)-\int_{\mathbb{R}} f(y) W_{0}(d y) \\
& =\int_{0}^{t}\left(\int_{\mathbb{R}} \frac{1}{2} \sigma^{2} f^{\prime \prime}(y) \widetilde{W}_{s}(d y)\right) d s+\int_{0}^{t}\left(\int_{\mathbb{R}} V(s, y) f^{\prime}(y) \widetilde{W}_{s}(d y)\right) d s
\end{array}
$$

where $V(t, x)=\int_{\mathbb{R}} H(x-y) \widetilde{W}_{t}(d y)$

Observe that $\widetilde{W}_{t}$ has a density $\widetilde{\gamma}_{t}(\cdot)$ for all $t>0$, given by

$$
\widetilde{\gamma}_{t}(x)=\int_{\mathbb{R}} \widetilde{p}_{t}\left(x_{0}, x\right) W_{0}\left(d x_{0}\right)
$$

As $P_{t}^{\varepsilon}\left(x_{0}, \cdot\right)$ converges weakly to $\widetilde{P}_{t}\left(x_{0}, \cdot\right)$, it follows that

$$
\lim _{\varepsilon \rightarrow 0} \int_{\mathbb{R}} f(y) W_{t}^{\varepsilon}(d y)=\int_{\mathbb{R}} f(y) \widetilde{W}_{t}(d y)
$$

and

$$
\lim _{\varepsilon \rightarrow 0} \int_{\mathbb{R}} \frac{1}{2} \sigma^{2} f^{\prime \prime}(y) W_{t}^{\varepsilon}(d y)=\int_{\mathbb{R}} \frac{1}{2} \sigma^{2} f^{\prime \prime}(y) \widetilde{W_{t}}(d y)
$$

Since $W_{t}^{\varepsilon}$ solves (3.5), to obtain (3.7), it simply remains to prove that

$$
\lim _{\varepsilon \rightarrow 0} \int_{0}^{t}\left(\int_{\mathbb{R}} V^{\varepsilon}(s, y) f^{\prime}(y) W_{s}^{\varepsilon}(d y)-\int_{\mathbb{R}} V(s, y) f^{\prime}(y) \widetilde{W}_{s}(d y)\right) d s=0 .
$$


We estimate

$$
\begin{gathered}
\int_{0}^{t}\left|\int_{\mathbb{R}} V^{\varepsilon}(s, y) f^{\prime}(y) W_{s}^{\varepsilon}(d y)-\int_{\mathbb{R}} V(s, y) f^{\prime}(y) \widetilde{W}_{s}(d y)\right| d s \\
\leq \int_{0}^{t}\left|\int_{\mathbb{R}}\left(V^{\varepsilon}(s, y)-V(s, y)\right) f^{\prime}(y) W_{s}^{\varepsilon}(d y)\right| d s \\
\quad+\int_{0}^{t}\left|\int_{\mathbb{R}} V(s, y) f^{\prime}(y)\left(W_{s}^{\varepsilon}(d y)-\widetilde{W}_{s}(d y)\right)\right| d s .
\end{gathered}
$$

For all $t>0$, we have that $V(t, \cdot)$ is the distribution function of a finite measure which is absolutely continuous with respect to Lebesgue measure; thus, $V(t, \cdot)$ is a bounded continuous function, from which we deduce the convergence towards 0 of the second term of the right-hand side when $\varepsilon$ tends to 0 .

Denote by $F$ the first term of the right-hand side. We have

$$
F \leq \int_{\mathbb{R}}\left[\int_{0}^{t}\left|\int_{\mathbb{R}}\left(V^{\varepsilon}(s, y)-V(s, y)\right) f^{\prime}(y) p_{s}^{\varepsilon}\left(x_{0}, y\right) d y\right| d s\right] W_{0}\left(d x_{0}\right) .
$$

As $p_{s}^{\varepsilon}\left(x_{0}, \cdot\right)$ belongs to $L^{2}(\mathbb{R})$ for all $s>0$, we get from $(3.6)$

$$
F \leq C \int_{0}^{t} \sqrt{\int_{\mathbb{R}}\left(V^{\varepsilon}(s, y)-V(s, y)\right)^{2} f^{\prime 2}(y) d y} \frac{1}{s^{\frac{1}{4}}} d s .
$$

By definition of $V^{\varepsilon}(s, \cdot)$ and $V(s, \cdot)$, we have that

$$
\begin{aligned}
\int_{\mathbb{R}}\left(V^{\varepsilon}(s, y)-V(s, y)\right)^{2} f^{\prime 2}(y) d y \\
=\int_{\mathbb{R}}\left(\int_{\mathbb{R}} H^{\varepsilon}(z-y) W_{s}^{\varepsilon}(d z)-\int_{\mathbb{R}} H(y-z) \widetilde{W}_{s}(d z)\right)^{2} f^{\prime 2}(y) d y \\
\leq \int_{\mathbb{R}} 2\left(\int_{\mathbb{R}}\left(H^{\varepsilon}(y-z)-H(y-z)\right) W_{s}^{\varepsilon}(d z)\right)^{2} f^{\prime 2}(y) d y \\
\quad+\int_{\mathbb{R}} 2\left(\int_{\mathbb{R}} H(y-z)\left(W_{s}^{\varepsilon}(d z)-\widetilde{W}_{s}(d z)\right)\right)^{2} f^{\prime 2}(y) d y .
\end{aligned}
$$

Therefore,

$$
\begin{aligned}
F \leq & A \int_{0}^{t} \sqrt{\int_{\mathbb{R}}\left(\int_{\mathbb{R}}\left|H^{\varepsilon}(y-z)-H(y-z)\right| d z\right) f^{\prime 2}(y) d y} \frac{C}{\sqrt{s}} d s \\
& +A \int_{0}^{t} \sqrt{\int_{\mathbb{R}}\left(\int_{-\infty}^{y} W_{s}^{\varepsilon}(d z)-\int_{-\infty}^{y} \widetilde{W}_{s}(d z)\right)^{2} f^{\prime 2}(y) d y} \frac{C}{s^{\frac{1}{4}}} d s .
\end{aligned}
$$

One easily sees that for all $y$ in $\mathbb{R}$,

$$
\left(\int_{\mathbb{R}}\left|\left(H * \Phi_{\varepsilon}\right)(y-z)-H(y-z)\right| d z\right) \leq \frac{2 \varepsilon}{\sqrt{2 \pi}} .
$$

Moreover, as $W_{s}^{\varepsilon}$ and $\widetilde{W}_{s}$ are absolutely continuous with respect to Lebesgue measure, the term $\left(\int_{-\infty}^{y} W_{s}^{\varepsilon}(d z)-\int_{-\infty}^{y} \widetilde{W}_{s}(d z)\right)$ tends to 0 for all $y$ in $\mathbb{R}$. Now it remains to use the bounded convergence theorem (note that $f^{\prime 2}$ is integrable on $\mathbb{R}$ 
since it is continuous and it has compact support; also note that $\left(\frac{1}{\sqrt{s}}\right)$ and $\left(\frac{1}{s^{\frac{1}{4}}}\right)$ are integrable on $[0, t]): F$ tends to 0 when $\varepsilon$ tends to 0 , which proves $(3.8)$.

We are now in position to complete the proof of Theorem 3.1.

As $V$ is bounded on $[0, T] \times \mathbb{R}$, the stochastic differential equation

$$
d z_{t}=V\left(t, z_{t}\right) d t+\sigma d w_{t}, t \in[0, T]
$$

has a unique solution in the sense of probability law (see Karatzas and Shreve [15] e.g.).

We now show that for all Borel sets $\mathcal{A} \subset \mathbb{R}$ we have

$$
\widetilde{W}_{t}(\mathcal{A})=\int_{\mathbb{R}} P_{t}\left(x_{0}, \mathcal{A}\right) W_{0}\left(d x_{0}\right),
$$

where $P_{t}\left(x_{0}, \cdot\right)$ is the transition probability of $\left(z_{t}\right)$.

For any $x_{0}$ in $\mathbb{R}$, consider the process $\left(z_{t}\left(x_{0}\right)\right)$, solution to $(3.9)$ and emanating from $x_{0}$ at time $t=0$. For all $t \in(0, T]$, the law of $z_{t}\left(x_{0}\right)$ has a density, denoted by $p_{t}\left(x_{0}, \cdot\right)$; define the function $\gamma_{t}(\cdot)$ on $\mathbb{R}$ by

$$
\gamma_{t}(x)=\int_{\mathbb{R}} p_{t}\left(x_{0}, \cdot\right) W_{0}\left(d x_{0}\right)
$$

We now show that the density $\widetilde{\gamma}_{t}(\cdot)$ of $\widetilde{W}_{t}$ is equal to $\gamma_{t}(\cdot)$.

Consider the equation

$$
q_{t}=S_{t} W_{0}-\int_{0}^{t} S_{t-s}\left(\frac{\partial}{\partial x}\left(q_{s} V(s, \cdot)\right)\right) d s, \forall t \in(0, T],
$$

where $S_{t}$ is the semigroup defined by $S_{t} U=g_{t} * U$ and $g_{t}$ is the density of the law of $\sigma w_{t}$.

\section{Lemma 3.4.}

(i) For any $x_{0}$ in $\mathbb{R}$, for any solution in the sense of probability law $\left(z_{t}\left(x_{0}\right)\right)$ of (3.9), the corresponding function $\gamma_{t}$ is a weak solution of (3.11) .

(ii) There exists at most one function $q_{t}$ in $L^{1}(\mathbb{R})$ which is a weak solution of (3.11).

Proof. We first prove (i).

Fix $t$ in $(0, t]$ and $f$ in $C^{\infty}(\mathbb{R})$ with compact support, and set

$$
G(s, x)=S_{t-s} f(x) \text { for } 0 \leq s<t .
$$

The function $G(s, x)$ solves

$$
\left\{\begin{array}{l}
\frac{\partial G}{\partial s}+\frac{1}{2} \sigma^{2} \frac{\partial^{2} G}{\partial x^{2}}=0,0 \leq s<t \\
G(t, x)=f(x)
\end{array}\right.
$$

Itô's formula shows that

$$
G\left(t, z_{t}\left(x_{0}\right)\right)=G\left(0, x_{0}\right)+\int_{0}^{t} \frac{\partial G}{\partial x}\left(s, z_{s}\left(x_{0}\right)\right) d w_{s}+\int_{0}^{t} \frac{\partial G}{\partial x}\left(s, z_{s}\left(x_{0}\right)\right) V\left(s, z_{s}\left(x_{0}\right)\right) d s
$$

from which

$$
\int_{\mathbb{R}} f(x) \gamma_{t}(x) d x=\int_{\mathbb{R}} G(0, x) W_{0}(d x)+\int_{0}^{t} \int_{\mathbb{R}} \frac{\partial}{\partial x} G(s, x) V(s, x) \gamma_{s}(x) d x d s .
$$

One can easily deduce that $\gamma_{t}$ solves (3.11) in the weak sense. 
We now prove (ii). Let $u_{t}$ and $v_{t}$ be two weak solutions of (3.11) belonging to $L^{1}(\mathbb{R})$; then, for all $t \in(0, T]$, one has

$$
\begin{aligned}
\left\|u_{t}-v_{t}\right\|_{L^{1}(\mathbb{R})} & =\left\|\int_{0}^{t} S_{t-s} \frac{\partial}{\partial x}\left\{\left(u_{s}(x)-v_{s}(x)\right) V(s, x)\right\}\right\|_{L^{1}(\mathbb{R})} \\
& \leq \int_{0}^{t}\left\|g_{t-s} * \frac{\partial}{\partial x}\left\{\left(u_{s}(x)-v_{s}(x)\right) V(s, x)\right\}\right\|_{L^{1}(\mathbb{R})} d s \\
& \leq \int_{0}^{t}\left\|\frac{\partial}{\partial x} g_{t-s}\right\|_{L^{1}(\mathbb{R})} \times A\left\|u_{s}(x)-v_{s}(x)\right\|_{L^{1}(\mathbb{R})} d s \\
& \leq \int_{0}^{t} \frac{2 A}{\sqrt{2 \pi(t-s) \sigma^{2}}}\left\|u_{s}(x)-v_{s}(x)\right\|_{L^{1}(\mathbb{R})} d s .
\end{aligned}
$$

Noting that $s \rightarrow 1 / \sqrt{t-s}$ is integrable on $[0, t]$, one applies Gronwall's lemma to conclude.

To show that $\widetilde{\gamma}_{t}(\cdot)$ and $\gamma_{t}(\cdot)$ are equal, it now remains to prove that $\widetilde{\gamma}_{t}(\cdot)$ is also a solution to (3.11) in the weak sense. As $\widetilde{\gamma}_{t}(\cdot)$ is the density of $\widetilde{W}_{t}$, it satisfies (3.3), and an easy computation shows

$$
\int_{\mathbb{R}} f(x) \widetilde{\gamma}_{t}(x) d x=\int_{\mathbb{R}} G(0, x) W_{0}(d x)+\int_{0}^{t} \int_{\mathbb{R}} \frac{\partial}{\partial x} G(s, x) V(s, x) \widetilde{\gamma}_{s}(x) d x d s .
$$

Then one can show that $\widetilde{\gamma}_{t}$ is a solution in the weak sense of (3.11), which proves (3.10). Thus, we have proven part (a) of Theorem 3.1.

For part (b), see our paper [3] for the proof when the initial condition is monotonic and M. Bossy's thesis [2] for the extension to the present situation.

3.4. The algorithm and its convergence rate. We have obtained that the solution of the Burgers equation with the initial condition $V_{0}(x)=\int_{-\infty}^{x} W_{0}(d y)$, where $W_{0} \in \mathcal{M}\left(\mathbb{R} ; a^{+} ; a^{-}\right)$, satisfies

$$
V(t, x)=a^{+} \mathbb{E}_{U_{0}^{+}} H\left(x-z_{t}\right)-a^{-} \mathbb{E}_{U_{0}^{-}} H\left(x-z_{t}\right) ;
$$

here the process $\left(z_{t}\right)$ satisfies

$$
d z_{t}=V\left(t, z_{t}\right) d t+\sigma d w_{t}
$$

and $U_{0}^{+}$and $U_{0}^{-}$are such that

$$
W_{0}=a^{+} U_{0}^{+}-a^{-} U_{0}^{-} .
$$

This probabilistic representation of the solution allows us to adapt the approximation method developed for smooth interaction kernels.

In the sequel we assume:

$\left(\mathrm{H}^{\prime}\right)$ The positive and negative parts, $U_{0}^{+}$and $U_{0}^{-}$, of $W_{0}$ satisfy :

(i) either $U_{0}^{ \pm}$is the Dirac measure at $x_{0}$, or

(ii) $U_{0}^{ \pm}$has a smooth density $u_{0}^{ \pm}$satisfying one of the two conditions:

- $u_{0}^{ \pm}(\cdot)$ is a continuous and bounded function and there exist strictly positive constants $M, \eta$ and $\alpha$ such that for all $|x|>M, u_{0}^{ \pm}(x) \leq$ $\eta \exp \left(-\alpha \frac{x^{2}}{2}\right)$ 
- $u_{0}^{ \pm}$is a function with compact support and is continuous on this support.

Let $N$ be an even number of particles. We denote by $y_{0}^{i}$ for $i=1, \ldots, N$ the initial locations of the particles. The $N / 2$ first (respectively last) locations are chosen in order to approximate the distribution function of the probability measure $U_{0}^{+}$(respectively $U_{0}^{-}$) by a piecewise constant function: if $U_{0}^{ \pm}$is a Dirac mass at point $x_{0}$, we locate the corresponding particles at $x_{0}$; if $U_{0}^{ \pm}$has a density function, we invert its distribution function (see $\S 2$ ). Thus, the piecewise constant function

$$
\bar{V}_{0}(x):=\frac{1}{N} \sum_{i=1}^{N} A_{i} H\left(x-y_{0}^{i}\right)
$$

with

$$
\left\{\begin{array}{l}
A_{i}=2 a^{+}, \quad i=1, \ldots, N / 2, \\
A_{i}=-2 a^{-}, \quad i=N / 2+1, \ldots, N,
\end{array}\right.
$$

approximates $V_{0}$ and (cf. above) the initialization error $\left\|V_{0}(\cdot)-\bar{V}_{0}(\cdot)\right\|_{L^{1}(\mathbb{R})}$ is of $\operatorname{order} \mathcal{O}\left(\frac{1}{N} \sqrt{\log (N)}\right)$.

Instead of identical weights equal to $\frac{1}{N}$, the particles now have different weights fixed at time 0 . At each discretization time $t_{k}$, we approximate the solution $V\left(t_{k}, \cdot\right)$ by

$$
\bar{V}_{t_{k}}(x)=\frac{1}{N} \sum_{i=1}^{N} A_{i} H\left(x-Y_{t_{k}}^{i}\right),
$$

where the $\left(Y_{t_{k}}^{i}\right)$ 's are defined by

$$
\left\{\begin{aligned}
Y_{t_{k+1}}^{i} & =Y_{t_{k}}^{i}+\bar{V}_{t_{k}}\left(Y_{t_{k}}^{i}\right) \Delta t+\sigma\left(w_{t_{k+1}}^{i}-w_{t_{k}}^{i}\right) \\
& =Y_{t_{k}}^{i}+\frac{1}{N} \sum_{j=1}^{N} A_{j} H\left(Y_{t_{k}}^{i}-Y_{t_{k}}^{j}\right) \Delta t+\sigma\left(w_{t_{k+1}}^{i}-w_{t_{k}}^{i}\right), \\
Y_{0}^{i}= & y_{0}^{i} .
\end{aligned}\right.
$$

The rate of convergence is the same as in the case of monotonic initial conditions (the proof given in Bossy and Talay [3] needs only a few modifications consisting in bounding the $A_{i}$ 's from above by a constant):

Theorem 3.5. With $T$ fixed, let $\Delta t>0$ be such that $T=\Delta t K, K \in \mathbb{N}$.

Let $V\left(t_{k}, x\right)$ be the solution at time $t_{k}=k \Delta t$ of the Burgers equation with the initial condition $V_{0}$, and let $\bar{V}_{t_{k}}(x)$ be defined as above.

Under $\left(\mathrm{H}^{\prime}\right)$, there exists a strictly positive constant $C$, depending on $\sigma, W_{0}$ and $T$ but uniform in $N$ and $\Delta t$, such that, for all $k \in\{1, \ldots, K\}$

$$
\mathbb{E}\left\|V\left(t_{k}, \cdot\right)-\bar{V}_{t_{k}}(\cdot)\right\|_{L^{1}(\mathbb{R})} \leq C\left(\left\|V_{0}-\bar{V}_{0}\right\|_{L^{1}(\mathbb{R})}+\frac{1}{\sqrt{N}}+\sqrt{\Delta t}\right) .
$$

\section{Numerical Results for the Burgers equation}

In order to compare our theoretical bounds with the real performance of our method, we consider the Burgers equation with an initial condition for which the 
solution can be written in an explicit form. Our experiments show that our estimates are not crude and illustrate the good behavior of the algorithm when the diffusion coefficient tends to zero.

For any initial condition $V_{0}$ such that

$$
\int_{0}^{x} V_{0}(y) d y=\mathcal{O}(x)
$$

the solution of the Burgers equation has the following form (cf. Hopf [14])

$$
V(t, x)=\frac{\int_{\mathbb{R}} V_{0}(y) \exp \left(-\frac{1}{\sigma^{2}}\left[\frac{(x-y)^{2}}{2 t}+\int_{0}^{y} V_{0}(z) d z\right]\right) d y}{\int_{\mathbb{R}} \exp \left(-\frac{1}{\sigma^{2}}\left[\frac{(x-y)^{2}}{2 t}+\int_{0}^{y} V_{0}(z) d z\right]\right) d y},(t, x) \in[0, T] \times \mathbb{R} .
$$

We choose

$$
V_{0}(x)=1-H(x),
$$

where $H$ is the Heaviside function. In that case the above expression can be numerically approximated with good accuracy; the exact value is

$$
V(t, x)=1-\frac{\operatorname{erfc}\left(\frac{-x}{\sqrt{2 \sigma^{2} t}}\right)}{\operatorname{erfc}\left(\frac{-x}{\sqrt{2 \sigma^{2} t}}\right)+\exp \left(\frac{t-2 x}{2 \sigma^{2}}\right)\left(2-\operatorname{erfc}\left(\frac{t-x}{\sqrt{2 \sigma^{2} t}}\right)\right)},
$$

where the function erfc is defined by

$$
\operatorname{erfc} x=\frac{2}{\sqrt{\pi}} \int_{x}^{+\infty} \exp \left(-u^{2}\right) d u .
$$

As $1-V_{0}$ is the "cumulative function of the Dirac mass centered at zero", the initialization of the particles is very simple: we just place the $N$ particles at zero.

Figure 1 shows the general shape of the solution of our test problem: it is a travelling wave with speed $1 / 2$. The discontinuity of $V_{0}$ immediately disappears under the effect of the diffusion term of the equation, and for all $t>0$ the solution propagates without any deformation.

We are interested in the dependence of the error $\mathbb{E}\left\|V(T, \cdot)-\bar{V}_{T}(\cdot)\right\|_{L^{1}(\mathbb{R})}$ on the two parameters of the algorithm: the number of particles $N$ and the time step $\Delta t$.

For a fixed initialization $\rho$ of the random number generator, we compute an approximation of the $L^{1}$ norm of the error at time $T=K \Delta t$ with the discrete norm

$$
\left\|V(T, \cdot)-\bar{V}_{T}(\cdot)\right\|_{L^{1}(\mathbb{R})} \simeq \sum_{i=1}^{N}\left(Y_{T}^{i+1}-Y_{T}^{i}\right)\left|V\left(T, Y_{T}^{i}\right)-\bar{V}_{T}\left(Y_{T}^{i}\right)\right|:=\operatorname{Error}_{\rho}
$$

where the $\left(Y_{T}^{i}\right)$ are the positions of the particles arranged in nondecreasing order. When the interacting kernel is the Heaviside function, a very simple way to compute

$$
\left(\frac{1}{N} \sum_{j=1}^{N} H\left(Y_{t_{k}}^{i}-Y_{t_{k}}^{j}\right) \text { for all } i\right)
$$




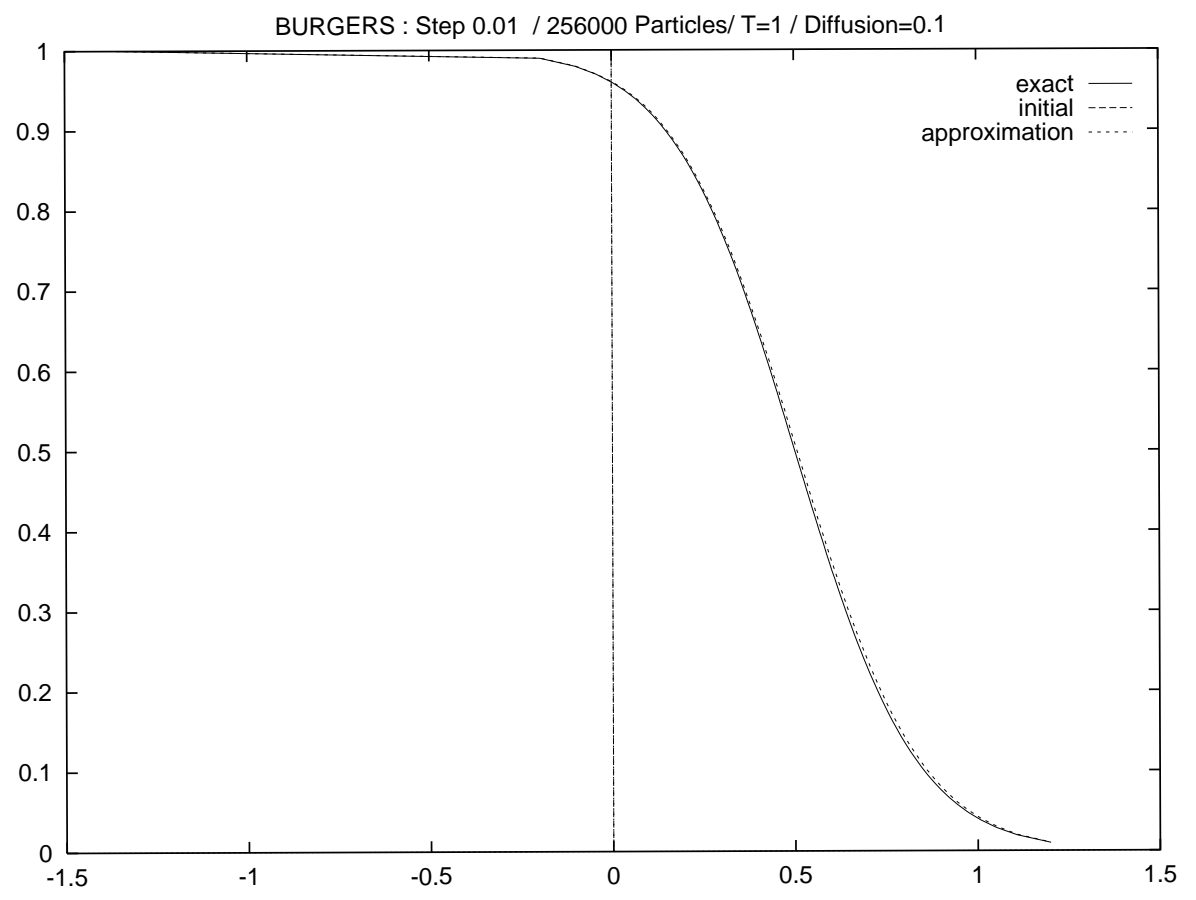

Figure 1. General shape of the solution

is to sort the positions at each time step. If the positions are sorted in nondecreasing order, at each time step and for each $i$ we must compute

$$
\bar{V}_{t_{k}}\left(Y_{t_{k}}^{i}\right)=\frac{1}{N} \sum_{j=1}^{N} H\left(Y_{t_{k}}^{i}-Y_{t_{k}}^{j}\right)=\frac{i}{N}, \forall k=1, \ldots, N .
$$

We estimate the expectation of the error by an average based on 20 independent trials corresponding to different initializations of the random number generator; we also estimate the variance:

$$
\begin{aligned}
& \mathbb{E}\left\|V(T, \cdot)-\bar{V}_{T}(\cdot)\right\|_{L^{1}(\mathbb{R})} \simeq \frac{1}{20} \sum_{\rho=1}^{20} \text { Error }_{\rho}:=\overline{\mathbb{E}} \text { Error }, \\
& \operatorname{Var}\left\|V(T, \cdot)-\bar{V}_{T}(\cdot)\right\|_{L^{1}(\mathbb{R})} \simeq \frac{1}{20} \sum_{\rho=1}^{20}\left(\text { Error }_{\rho}-\overline{\mathbb{E}} \text { Error }\right)^{2} .
\end{aligned}
$$

Dependence on $N$. Table 1 presents numerical estimates of the $L^{1}$ norm of the error, computed as described above. We study the dependence of the error on $N$ as follows: $\Delta t$ is fixed, small enough to only observe the effect of $N$ on the error, and we give the results correponding to $N=250 \times 4^{k}$; we observe that changing $k$ to $k+1$ induces a division by 2 of the expectation and of the standard deviation of the error, which confirms the order $\mathcal{O}(1 / \sqrt{N})$. 
TABle 1. Dependence on $N$ of the error; $\frac{1}{2} \sigma^{2}=0.1, T=1$

Estimation of the expectation of the error in the $L^{1}$ norm

\begin{tabular}{|c||c|c|c|c|c|c|}
\hline \multicolumn{1}{|c||}{} & \multicolumn{6}{c|}{ Number of particles } \\
\hline$\Delta t$ & 250 & 1000 & 4000 & 16000 & 64000 & 256000 \\
\hline \hline 0.01 & $3.194 .10^{-2}$ & $1.474 .10^{-2}$ & $6.949 .10^{-3}$ & $3.484 .10^{-3}$ & $2.097 .10^{-3}$ & $1.899 .10^{-3}$ \\
\hline 0.005 & $2.959 .10^{-2}$ & $1.199 .10^{-2}$ & $6.699 .10^{-3}$ & $3.845 .10^{-3}$ & $1.993 .10^{-3}$ & $1.083 .10^{-3}$ \\
\hline
\end{tabular}

Estimation of the standard deviation of the error in the $L^{1}$ norm

\begin{tabular}{|c||c|c|c|c|c|c|}
\hline \multicolumn{1}{|c||}{} & \multicolumn{6}{c|}{ Number of particles } \\
\hline$\Delta t$ & 250 & 1000 & 4000 & 16000 & 64000 & 256000 \\
\hline \hline 0.01 & $3.458 .10^{-2}$ & $1.685 .10^{-2}$ & $7.42 .10^{-3}$ & $3.674 .10^{-3}$ & $2.230 .10^{-3}$ & $1.615 .10^{-3}$ \\
\hline 0.005 & $3.231 .10^{-2}$ & $1.299 .10^{-2}$ & $6.889 .10^{-3}$ & $3.979 .10^{-3}$ & $2.087 .10^{-3}$ & $1.155 .10^{-3}$ \\
\hline
\end{tabular}

TABLE 2. Dependence on $\Delta t$ of the error

Estimation of the $L^{1}$ norm of the error; $N=100000,1 / 2 \sigma^{2}=0.1, T=1$

\begin{tabular}{|c||c||c||c|}
\hline$\Delta t$ & Expectation & Variance & S. D. \\
\hline \hline $1 / 2$ & $7.9666 .10^{-2}$ & $4.1151 .10^{-7}$ & $6.4149 .10^{-4}$ \\
\hline $1 / 4$ & $3.5357 .10^{-2}$ & $3.4869 .10^{-7}$ & $5.9050 .10^{-4}$ \\
\hline $1 / 8$ & $1.6719 .10^{-2}$ & $5.1327 .10^{-7}$ & $7.1643 .10^{-4}$ \\
\hline $1 / 16$ & $8.3335 .10^{-3}$ & $3.2875 .10^{-7}$ & $5.7337 .10^{-4}$ \\
\hline $1 / 32$ & $4.1286 .10^{-3}$ & $4.7058 .10^{-7}$ & $6.8599 .10^{-4}$ \\
\hline $1 / 64$ & $2.7924 .10^{-3}$ & $6.0759 .10^{-7}$ & $7.7948 .10^{-4}$ \\
\hline $1 / 128$ & $2.1373 .10^{-3}$ & $6.6170 .10^{-7}$ & $8.1345 .10^{-4}$ \\
\hline $1 / 256$ & $1.6634 .10^{-3}$ & $3.5202 .10^{-7}$ & $5.9331 .10^{-4}$ \\
\hline $1 / 512$ & $1.5902 .10^{-3}$ & $2.3290 .10^{-7}$ & $4.8260 .10^{-4}$ \\
\hline
\end{tabular}

Dependence on $\Delta t$. We apply the same strategy to study the dependence of the error on $\Delta t$. Table 2 presents numerical estimates of the expectation, the variance and the standard deviation of the $L^{1}$ norm of the error. We fix the number of particles sufficiently large to neglect the effect of $N$ in the error. When the time step $\Delta t$ is successively divided by 2 , from $\Delta t=1 / 2$ to $\Delta t=1 / 128$, the expectation of the error is also divided by 2 . From $\Delta t=128$ to $\Delta t=1 / 512$ the error seems constant because for so small discretization steps the effect of $N$ cannot be neglected unless $N$ is extremely large. Thus the order of convergence in $\Delta t$ seems better than predicted by our theoretical estimate: $\Delta t$ rather than $\sqrt{\Delta t}$. In our theoretical analysis, to bound the error caused by the time discretization, we used the rate of convergence in $L^{2}(\Omega)$ of the Euler scheme for the SDE satisfied by $\left(z_{t}\right)$; this bound is too crude and likely hides an additional averaging effect of the large number of particles. 
TABLE 3. Dependence on $\sigma$ of the error

Estimation of the $L^{1}$ norm of the error; $N=100000, T=1, \Delta t=0.01$

\begin{tabular}{|c||c||c||c|}
\hline$\frac{1}{2} \sigma^{2}$ & Expectation & Variance & S. D. \\
\hline \hline 0.001 & $1.2266 .10^{-3}$ & $3.0045 .10^{-10}$ & $1.7333 .10^{-5}$ \\
\hline 0.01 & $1.3396 .10^{-3}$ & $1.8990 .10^{-8}$ & $1.3780 .10^{-4}$ \\
\hline 0.1 & $2.2841 .10^{-3}$ & $7.2588 .10^{-7}$ & $8.5198 .10^{-4}$ \\
\hline 1 & $6.6735 .10^{-3}$ & $8.3870 .10^{-6}$ & $2.8960 .10^{-3}$ \\
\hline 10 & $2.1060 .10^{-2}$ & $8.3495 .10^{-5}$ & $9.1376 .10^{-3}$ \\
\hline 100 & $6.6778 .10^{-2}$ & $8.2950 .10^{-4}$ & $2.8801 .10^{-2}$ \\
\hline 1000 & 0.2114 & $8.2611 .10^{-3}$ & $9.0890 .10^{-2}$ \\
\hline
\end{tabular}

Remark 4.1. As we expected from our theoretical bounds, there is no need to correlate $\Delta t$ with $N$ in order to observe the convergence.

Moreover, we only have presented here results for the discrete $L^{1}$ norm, but the numerical rate of convergence for the discrete $L^{\infty}$ norm is similar.

Behavior of the algorithm with respect to the diffusion coefficient. In our theoretical bounds, the dependence on $\sigma$ does not appear explicitly but the effect of this parameter in the convergence is very important. Table 3 presents numerical results for various values of $\sigma$, the values of $N$ and $\Delta t$ being fixed. As $\sigma$ decreases, the expectation and the variance of the error become smaller; this is due to the fact that the particles tend to spread less when the diffusion coefficient decreases, which improves the precision. The good behavior of the algorithm as $\sigma$ tends to 0 makes us believe that it could be used for the approximation of the solution of the inviscid Burgers equation: the diffusion term added to the equation must then be seen as an artificial viscosity term which permits the algorithm to operate. It is well known that the solution $V_{\varepsilon}$ of the perturbed problem

$$
\left\{\begin{array}{l}
\frac{\partial V_{\varepsilon}}{\partial t}+V_{\varepsilon} \frac{\partial V_{\varepsilon}}{\partial x}=\varepsilon \frac{\partial^{2} V_{\varepsilon}}{\partial x^{2}} \text { in }(0, T] \times \mathbb{R}, \\
V_{\varepsilon}(0, x)=V_{0}(x)
\end{array}\right.
$$

converges to the entropic solution of the inviscid Burgers equation

$$
\left\{\begin{array}{l}
\frac{\partial V}{\partial t}+V \frac{\partial V}{\partial x}=0 \text { in }(0, T] \times \mathbb{R}, \\
V(0, x)=V_{0}(x)
\end{array}\right.
$$

(see, for example, S. N. Kružkov [16]); thus, one can expect a convergence result for the particle method when $\varepsilon$ tends to 0 ; we have not obtained such a result, but we illustrate it by studying the Riemann problem. In that case the initial condition $V_{0}(x)=1-H(x)$ corresponds to a shock located at the origin and the shock is stable; the solution of the inviscid Burgers equation is a shock wave which propagates with a constant speed equal to $1 / 2$. Figure 2 shows the approximation of the shock wave by the particle method with a diffusion coefficient equal to $10^{-7}$. 


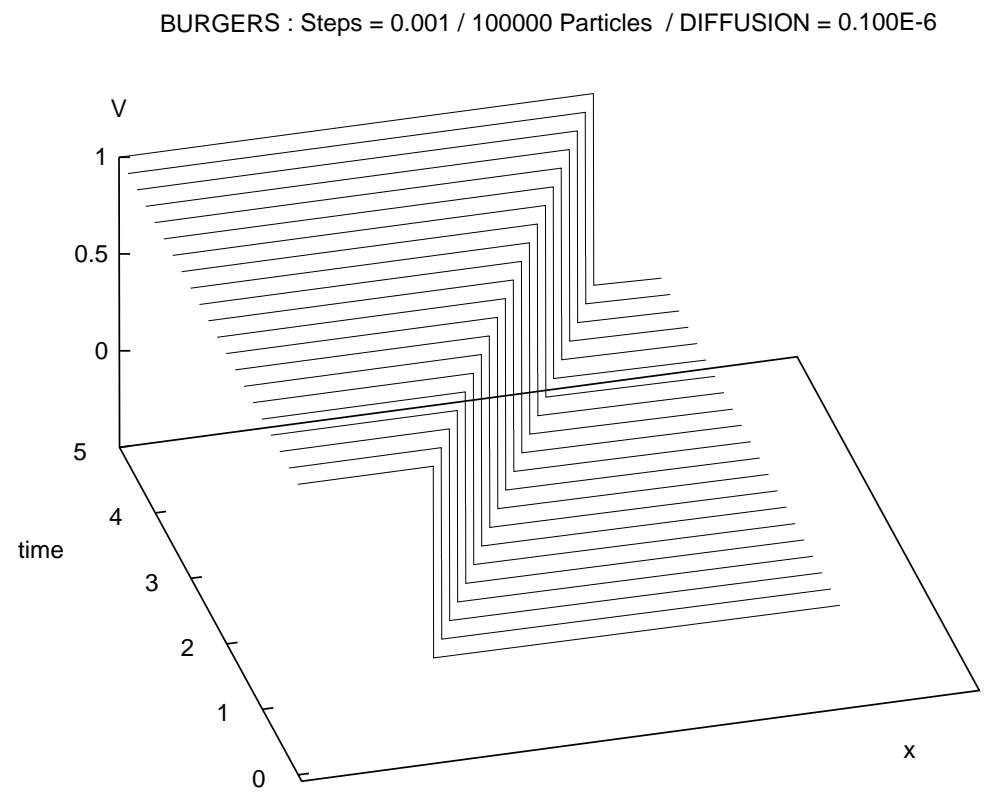

FiguRE 2. Shock wave, $T=0$ to $T=5$

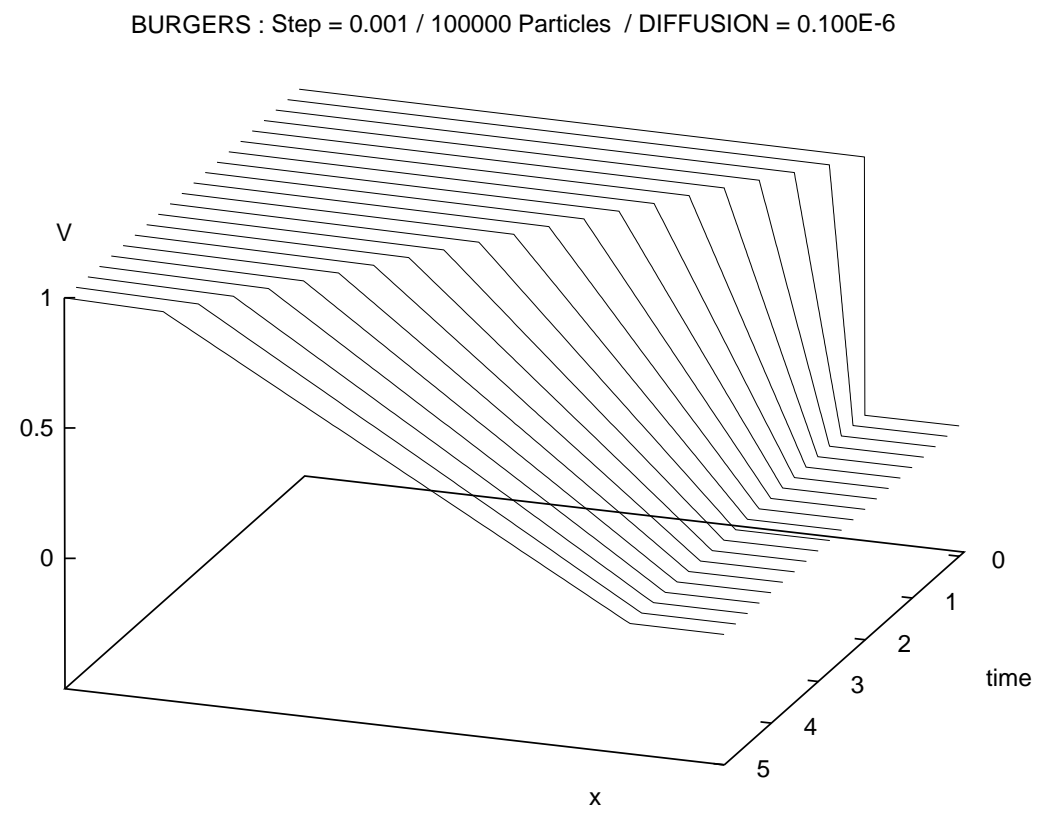

Figure 3. Expanding wave, $T=0$ to $T=5$ 
When the initial condition $V_{0}$ is equal to the Heaviside function, the initial shock is unstable. The discontinuity disappears immediately and an expanding wave propagates (see Figure 3).

\section{Concluding Remarks}

We have introduced a stochastic particle method to solve a McKean-Vlasov PDE in $[0, T] \times \mathbb{R}$ with Lipschitz kernels. This method provides approximations for the distribution function and the density of the measure solution to the McKean-Vlasov equation. Our method and our convergence rate results could easily be extended to a multidimensional state space (in this latter case, the distribution function is not given by a PDE).

Our algorithm, our analysis and our numerical experiments for the Burgers equation with a nonmonotonic initial condition are a preliminary step: our aim now is to treat the 2-D incompressible Navier-Stokes equation and to give new error estimates for Chorin's random vortex methods. Compared to the present work, the additional difficulty comes from the fact that the interaction kernel corresponding to the Navier-Stokes equation is singular at the origin.

\section{APPENDIX}

A.1. Proof of Lemma 2.6. We must bound the following expression:

$$
A:=\int_{\mathbb{R}} \sqrt{\frac{1}{N} \sum_{i=1}^{N} \int_{-\infty}^{x-y_{0}^{i}} \frac{1}{\sqrt{t}} \exp \left(-\frac{y^{2}}{2 \bar{\sigma}^{2} t}\right) d y \times \int_{x-y_{0}^{i}}^{+\infty} \frac{1}{\sqrt{t}} \exp \left(-\frac{y^{2}}{2 \bar{\sigma}^{2} t}\right)} d y
$$

Under the hypothesis (H3-i), $\mu_{0}$ is the Dirac measure at the point $x_{0}$; the particles at time 0 are initialized to $\left(y_{i}^{0}=x_{0}, \forall i=1, \ldots, N\right)$. Thus,

$$
A=\int_{\mathbb{R}} \sqrt{\int_{-\infty}^{x-x_{0}} \frac{1}{\sqrt{t}} \exp \left(-\frac{y^{2}}{2 \bar{\sigma}^{2} t}\right) d y \times \int_{x-x_{0}}^{+\infty} \frac{1}{\sqrt{t}} \exp \left(-\frac{y^{2}}{2 \bar{\sigma}^{2} t}\right) d y} d x .
$$

Applying the inequality (2.16), we obtain

$$
\int_{-\infty}^{x-x_{0}} \frac{1}{\sqrt{t}} \exp \left(-\frac{y^{2}}{2 \bar{\sigma}^{2} t}\right) d y \times \int_{x-x_{0}}^{+\infty} \frac{1}{\sqrt{t}} \exp \left(-\frac{y^{2}}{2 \bar{\sigma}^{2} t}\right) d y \leq C \bar{\sigma}^{2} \exp \left(-\frac{x^{2}}{2 \bar{\sigma}^{2} t}\right)
$$

Thus,

$$
A \leq C \bar{\sigma} \int_{\mathbb{R}} \exp \left(-\frac{x^{2}}{4 \bar{\sigma}^{2} t}\right) d x \leq C \bar{\sigma}^{2} \sqrt{t}
$$

Assume now (H3-ii): $\mu_{0}$ has a smooth density. Let $M$ be the constant appearing in (H3-ii). Decomposing the integral $A$ in three parts (from $-\infty$ to $-M$, from $-M$ 
to $M$ and from $M$ to $+\infty)$, we get

$$
\begin{aligned}
A \leq & \underbrace{\int_{-\infty}^{-M} \sqrt{\frac{1}{N} \sum_{i=1}^{N} \int_{-\infty}^{x-y_{0}^{i}} \frac{1}{\sqrt{t}} \exp \left(-\frac{y^{2}}{2 \bar{\sigma}^{2} t}\right) d y} d x}_{I^{-}}+C \\
& +\underbrace{\int_{M}^{+\infty} \sqrt{\frac{1}{N} \sum_{i=1}^{N} \int_{x-y_{0}^{i}}^{+\infty} \frac{1}{\sqrt{t}} \exp \left(-\frac{y^{2}}{2 \bar{\sigma}^{2} t}\right) d y} d x}_{I^{+}}
\end{aligned}
$$

We only treat $I^{-}$, since $I^{+}$can be treated by symmetry. By definition of the $y_{0}^{i}$, s, there holds

$$
I^{-} \leq \frac{1}{\sqrt{t}} \int_{-\infty}^{-M} \sqrt{\frac{1}{N}\left[\sum_{i=1}^{N-1} \int_{-\infty}^{x-V_{0}^{-1}\left(\frac{i}{N}\right)} \exp \left(-\frac{y^{2}}{2 \bar{\sigma}^{2} t}\right) d y+\int_{-\infty}^{x-V_{0}^{-1}\left(1-\frac{1}{2 N}\right)} \exp \left(-\frac{y^{2}}{2 \bar{\sigma}^{2} t}\right) d y\right]} d x .
$$

Let $\Psi$ be the function on $(0,1)$ defined by

$$
\Psi(\theta)=\int_{-\infty}^{x-V_{0}^{-1}(\theta)} \exp \left(-\frac{y^{2}}{2 \bar{\sigma}^{2} t}\right) d y .
$$

As $V_{0}$ is an increasing function, $\Psi$ is a decreasing function and

$$
\begin{aligned}
& \frac{1}{2 N} \sum_{i=1}^{N-1} \Psi\left(\frac{i}{N}\right) \leq \frac{1}{N} \sum_{i=1}^{N-1} \Psi\left(\frac{i}{N}\right) \leq \int_{0}^{\frac{N}{N-1}} \Psi(\theta) d \theta \\
& \frac{1}{2 N} \Psi\left(1-\frac{1}{2 N}\right) \leq \int_{\frac{N}{N-1}}^{1} \Psi(\theta) d \theta
\end{aligned}
$$

Thus,

$$
\begin{aligned}
I^{-} & \leq \frac{\sqrt{2}}{\sqrt{t}} \int_{-\infty}^{-M} \sqrt{\int_{0}^{1} \int_{-\infty}^{x-V_{0}^{-1}(\theta)} \exp \left(-\frac{y^{2}}{2 \bar{\sigma}^{2} t}\right)} d y d \theta \\
& \leq \frac{\sqrt{2}}{\sqrt{t}} \int_{-\infty}^{-M} \sqrt{\int_{\mathbb{R}} \exp \left(-\frac{(x-u)^{2}}{2 \bar{\sigma}^{2} t}\right) V_{0}(u) d u} d x .
\end{aligned}
$$

From (H4) and (2.16), one deduces

$$
\mathbf{1}_{[u \leq-M]} V_{0}(u)+\mathbf{1}_{[u \geq M]}\left(1-V_{0}(u)\right) \leq C \exp \left(-\alpha \frac{u^{2}}{2}\right),
$$

which completes the proof.

A.2. Proof of Theorem 3.5. We follow arguments in [19] and only give a sketch of the proof.

Let $d$ be the Kantorovich-Rubinstein metric on $\mathcal{M}\left(\mathbb{R} ; a^{+} ; a^{-}\right)$:

$$
d(x, y)=\min (|x-y|, 1) .
$$

On the space $\mathcal{M}^{1}(\mathbb{R})=\mathcal{M}^{+}(\mathbb{R} ; 1)$, consider the metric

$$
R_{1}(\mu, \nu)=\inf _{P \in \mathbb{C}(\mu, \nu)} \int_{\mathbb{R}^{2}} P(d x, d y) d(x, y),
$$


where $\mathbb{C}(\mu, \nu)$ denotes the set of the probability laws on $\mathbb{R}^{2}$ whose one-dimensional distributions are $\mu$ and $\nu$. On $\mathcal{M}\left(\mathbb{R} ; a^{+} ; a^{-}\right)$, consider the metric

$$
\widehat{R}(\mu, \nu)=R_{1}\left(\frac{\mu^{+}}{a^{+}}, \frac{\nu^{+}}{a^{+}}\right)+R_{1}\left(\frac{\mu^{-}}{a^{-}}, \frac{\nu^{-}}{a^{-}}\right),
$$

where $\mu^{+}$and $\mu^{-}$(resp. $\nu^{+}$and $\nu^{-}$) are the positive parts of the Jordan decomposition of $\mu$ (resp. $\nu)$. The topology induced by $\widehat{R}$ is equivalent to the topology induced by the weak convergence and makes $\mathcal{M}\left(\mathbb{R} ; a^{+} ; a^{-}\right)$separable and complete (see Dobrushin [7] e.g.).

Let $C\left([0, T] ; \mathcal{M}\left(\mathbb{R} ; a^{+} ; a^{-}\right)\right)$be the space of continuous functions $\widetilde{\mu}:[0, T] \ni$ $t \rightarrow \widetilde{\mu}_{t} \in \mathcal{M}\left(\mathbb{R} ; a^{+} ; a^{-}\right) ;$this space is complete for the metric

$$
R_{T}\left(\widetilde{\mu}^{1}, \widetilde{\mu}^{2}\right)=\sup _{t \in[0, T]} \widehat{R}\left(\widetilde{\mu}_{t}^{1}, \widetilde{\mu}_{t}^{2}\right) .
$$

Let $P_{t}^{\tilde{\mu}}(\cdot, y)$ denote the probability transition of the process solution to

$$
d z_{t}^{\varepsilon}=\mathcal{V}^{\widetilde{\mu}}\left(t, z_{t}^{\varepsilon}\right) d t+\sigma d w_{t}, t \in[0, T],
$$

with

$$
\mathcal{V}^{\widetilde{\mu}}(t, x)=\int_{\mathbb{R}} H^{\varepsilon}(x-y) \widetilde{\mu}_{t}(d y) .
$$

Having fixed $W_{0}$, define the map

$$
\begin{aligned}
S\left(W_{0}\right): C\left([0, T] ; \mathcal{M}\left(\mathbb{R} ; a^{+} ; a^{-}\right)\right) & \longrightarrow C\left([0, T] ; \mathcal{M}\left(\mathbb{R} ; a^{+} ; a^{-}\right)\right), \\
\widetilde{\mu} & \longrightarrow S\left(W_{0}\right) \widetilde{\mu},
\end{aligned}
$$

where, for all Borel sets $\mathcal{A} \subset \mathbb{R}$,

$$
\left[S\left(W_{0}\right) \widetilde{\mu}\right]_{t}(A)=\int_{\mathbb{R}} P_{t}^{\widetilde{\mu}}(y, A) W_{0}(d y) .
$$

Itô's formula implies that $S\left(W_{0}\right) \widetilde{\mu}$ satisfies, for any function $f$ in $C_{K}^{2}(\mathbb{R})$,

$$
\left\{\begin{aligned}
\frac{d}{d t}\left(\int_{\mathbb{R}} f(y)\left[S\left(W_{0}\right) \widetilde{\mu}\right]_{t}(d y)\right)= & \left(\int_{\mathbb{R}} \frac{1}{2} \sigma^{2} f^{\prime \prime}(y)\left[S\left(W_{0}\right) \widetilde{\mu}\right]_{t}(d y)\right) \\
& +\left(\int_{\mathbb{R}} \mathcal{V}^{\widetilde{\mu}}(t, y) f^{\prime}(y)\left[S\left(W_{0}\right) \widetilde{\mu}\right]_{t}(d y)\right), \\
{\left[S\left(W_{0}\right) \widetilde{\mu}\right]_{0}=W_{0} . } &
\end{aligned}\right.
$$

Then, one can show that $S\left(W_{0}\right)$ is a contraction on $C\left([0, T] ; \mathcal{M}\left(\mathbb{R} ; a^{+} ; a^{-}\right)\right)$. From (A.1), one deduces that the fixed point of $S\left(W_{0}\right), W^{\varepsilon}=\left\{W_{t}^{\varepsilon}\right\}_{t \in[0, T]}$, is the unique solution of the regularized problem: see [19] for details.

\section{REFERENCES}

1. P. Bernard, D. Talay, and L. Tubaro. Rate of convergence of a stochastic particle method for the Kolmogorov equation with variable coefficients. Math. Comp., 63(208):555-587, 1994. MR 95c:65139

2. M. Bossy. Vitesse de Convergence d'Algorithmes Particulaires Stochastiques et Application à l'Equation de Burgers. PhD thesis, Université de Provence, 1995.

3. M. Bossy and D. Talay. Convergence rate for the approximation of the limit law of weakly interacting particles: application to the Burgers equation. Ann. Appl. Probab., To appear.

4. P. Cannarsa and V. Vespri. Generation of analytic semigroups by elliptic operators with unbounded coefficients. SIAM J. Math. Anal., 18:857-872, 1987. MR 88d:47053 
5. A.J. Chorin and J.E. Marsden. A Mathematical Introduction to Fluid Mechanics. Springer Verlag, New York, 1993. MR 94c:76002

6. A.L. Chorin. Vortex methods and Vortex Statistics - Lectures for Les Houches Summer School of Theoretical Physics. Lawrence Berkeley Laboratory Prepublications, 1993.

7. R.L. Dobrushin. Prescribing a system of random variables by conditional distributions. Theory Probab. Appl., 3:469, 1970.

8. A. Friedman. Stochastic Differential Equations and Applications, vol. 1. Academic Press, New York, 1975. MR 58:13350a

9. J. Gärtner. On the Mckean-Vlasov limit for interacting diffusions. Math. Nachr., 137:197-248, 1988. MR 90a:60184

10. J. Goodman. Convergence of the random vortex method. Comm. Pure Appl. Math., 40:189220, 1987. MR 88d:35159

11. K.E. Gustafson and J.A. Sethian (eds.). Vortex Methods and Vortex Motion. SIAM, 1991. MR 93f: 76078

12. O.H. Hald. Convergence of random methods for a reaction diffusion equation. SIAM J. Sci. Statist. Comput., 2:85-94, 1981. MR 83c:65210

13. Convergence of a random method with creation of vorticity. SIAM J. Sci. Statist. Comput., 7:1373-1386, 1986.

14. E. Hopf. The partial differential equation $u_{t}+u u_{x}=\mu u_{x x}$. Comm. Pure Appl. Math., 3:201230, 1950. MR 13:846c

15. I. Karatzas and S.E. Shreve. Brownian Motion and Stochastic Calculus. Springer-Verlag, New York, 1988. MR 89c:60096

16. S. N. Kružkov. First order quasilinear equation in several independent variables. Math. USSR Sbornik, 10:217-243, 1970. MR 42:2159

17. C. Léonard. Une loi des grands nombres pour des systèmes de diffusions avec interaction et à coefficients non bornés. Ann. Inst. Henri Poincaré, 22(2):237-262, 1986. MR 88f:60136

18. D.G. Long. Convergence of the random vortex method in two dimensions. J. Amer. Math. Soc., 1(4), 1988. MR 90a:65202

19. C. Marchioro and M. Pulvirenti. Hydrodynamics in two dimensions and vortex theory. Comm. Math. Phys., 84:483-503, 1982. MR 84e:35126

20. S. Méléard and S. Roelly-Coppoletta. A propagation of chaos result for a system of particles with moderate interaction. Stochastic Process. Appl., 26:317-332, 1987. MR 89e:60201

21. M. Métivier. Quelques problèmes liés aux systèmes infinis de particules et leurs limites. Lectures Notes in Math., 1204:426-446, 1984. MR 90h:60100

22. G.N. Milshtein. Approximate integration of stochastic differential equations. Theory Probab. Appl., 19:557-562, 1974.

23. K. Oelschläger. A martingale approach to the law of large numbers for weakly interacting stochastic processes. Ann. Probab., 12:458-479, 1984. MR 85j:60191

24. E.G. Puckett. A study of the vortex sheet method and its rate of convergence. SIAM J. Sci. Statist. Comput., 10(2):298-327, 1989. MR 90c:76016

25. Convergence of a random particle method to solutions of the Kolmogorov equation. Math. Comp., 52(186):615-645, 1989. MR 90h:65008

26. P.A. Raviart. An analysis of particle methods. In F. Brezzi, ed., Numerical Methods in Fluid Dynamics, vol. 1127 of Lecture Notes in Math., pp. 243-324. Springer-Verlag, Berlin, Heidelberg, New York, 1985. MR 87h:76010

27. S. Roberts. Convergence of a random walk method for the Burgers equation. Math. Comp., 52(186):647-673, 1989. MR 89i:65090

28. A.-S. Sznitman. Topics in propagation of chaos. In P.L. Hennequin, ed., Ecole d'Eté de Probabilités de Saint-Flour XIX - 1989, vol. 1464 of Lecture Notes in Math., pp. 165-251. SpringerVerlag, Berlin, Heidelberg, New York, 1991. MR 93b:60179

INRIA, 2004 Route des Lucioles, B.P. 93, 06902 Sophia-Antipolis Cedex, France

E-mail address: Mireille.Bossy@sophia.inria.fr

E-mail address: Denis.Talay@sophia.inria.fr 